\title{
Momentum space treatment of inclusive neutrino scattering off the deuteron and trinucleons
}

\author{
J. Golak, R. Skibiński, K. Topolnicki, H. Witała, and A. Grassi \\ M. Smoluchowski Institute of Physics, Jagiellonian University, PL-30348 Kraków, Poland \\ H. Kamada \\ Department of Physics, Faculty of Engineering, Kyushu Institute of Technology, Kitakyushu 804-8550, Japan \\ L. E. Marcucci \\ Department of Physics, University of Pisa, IT-56127 Pisa, Italy \\ and INFN-Pisa, IT-56127 Pisa, Italy
}

(Received 5 May 2018; revised manuscript received 1 June 2018; published 12 July 2018)

\begin{abstract}
Selected (anti)neutrino-induced reactions on ${ }^{2} \mathrm{H},{ }^{3} \mathrm{He}$, and ${ }^{3} \mathrm{H}$ are studied consistently in momentum space for (anti)neutrino energies up to $250 \mathrm{MeV}$. For most of these processes we provide predictions for the total cross sections, and in the case of the (anti)neutrino- ${ }^{3} \mathrm{He}$ and (anti)neutrino- ${ }^{3} \mathrm{H}$ inelastic scattering we compute examples of essential response functions, using the AV18 nucleon-nucleon potential and a single-nucleon weak current operator. For the reactions with the deuteron we comment on relativistic effects in the final-state kinematics and compare two-nucleon scattering states obtained in momentum and coordinate spaces. Our results from momentum space are compared with the theoretical predictions by G. Shen et al. [Phys. Rev. C 86, 035503 (2012)]. The observed disagreement can be attributed to the differences in kinematics and in the weak current operator.
\end{abstract}

DOI: 10.1103/PhysRevC.98.015501

\section{INTRODUCTION}

Neutrino scattering on nuclei has been studied for several decades. For information on the status of earlier theoretical treatments of neutrino-nucleus reactions, relevant to the detection of astrophysical neutrinos, we refer the reader to Ref. [1]. At the beginning of the century theoretical work was motivated by the establishment of the Sudbury Neutrino Observatory (SNO) and resulted in important predictions by Nakamura et al. $[2,3]$. They calculated cross sections for both charged-current(CC) and neutral-current- (NC) driven reactions, for incoming neutrino energies up to $150 \mathrm{MeV}$. The results of Ref. [2] and the bulk of predictions given in Ref. [3] were obtained within the "standard nuclear physics approach" [4], employing the AV18 nucleon-nucleon (NN) force [5] and supplementing the single-nucleon current with two-nucleon $(2 \mathrm{~N})$ current contributions related to this potential. Some calculations in Ref. [3] were done with the CD-Bonn NN potential [6] or using input from chiral effective field theory $(\chi \mathrm{EFT})$ in order to estimate theoretical uncertainties of the results, which were later used to analyze SNO experimental data [7].

More recent calculations by Shen et al. [8] were also done within the traditional approach, using the AV18 potential and corresponding nuclear weak current operators with a one-body part and two-body contributions, adjusted to the NN force. The authors of Ref. [8] studied inclusive neutrino scattering on the deuteron up to neutrino energies of $1 \mathrm{GeV}$ with configuration space methods. Although they introduced some changes in the $2 \mathrm{~N}$ current operator used by Nakamura et al., these modifications proved to be of minor importance and the results obtained by Shen et al. confirmed those of Nakamura et al. in the energy range up to $150 \mathrm{MeV}$. Conclusions presented in Ref. [8] provided important information on the role of $2 \mathrm{~N}$ currents and final-state interaction effects for the whole considered neutrino energy range, even though pion production channels were neglected.

Despite the successes achieved within the traditional approach, new calculations emerging from $\chi \mathrm{EFT}$ offered competitive results. In 2001 Butler et al. [9] studied the neutrinodeuteron break-up reactions at next-to-next-to-leading order (N2LO) in pionless $\chi$ EFT in the energy range up to $20 \mathrm{MeV}$. Their work agreed very well with the previous potential model calculations from Refs. [2,3].

Attempts to build a complete theoretical framework comprising consistent "chiral" $2 \mathrm{~N}$ and many-nucleon forces as well as electroweak current operators at a sufficiently high order of the chiral expansion have a long history. A construction of the chiral NN potential was pioneered by Weinberg $[10,11]$ almost 30 years ago and developed by several groups. In particular, Epelbaum et al. have prepared three generations of the chiral potentials. They started with the version of the NN potential, where the nonlocal regularization in momentum space was implemented [12-14]. They derived also the widely used chiral three-nucleon (3N) potential at N2LO [15], summarizing the work on chiral forces and their applications to processes involving few nucleons up to 2005 in Ref. [16]. Further important contributions from this group, partially reviewed in Ref. [17], dealt with the $3 \mathrm{~N}$ force at next-to-next-to-next-to-leading order (N3LO) [18,19], the four-nucleon force [20], and a formulation of the $\Delta$-full chiral perturbation theory [21,22].

The next generation of the chiral NN potential by Epelbaum et al. used a coordinate space regularization. This improved 
version from Refs. [23,24] led to a significant reduction of finite-cutoff artifacts, did not require any additional spectral function regularization, and directly employed low-energy constants determined from pion-nucleon scattering. These forces were used to study nucleon-deuteron scattering [25] and various electroweak processes in $2 \mathrm{~N}$ and $3 \mathrm{~N}$ systems [26].

The newest version of the Bochum-Bonn chiral NN potential, prepared up to fifth order in the chiral expansion (N4LO), was introduced very recently in Ref. [27]. Important changes include a removal of the redundant contact terms and regularization in momentum space, resulting in an excellent description of the proton-proton and neutron-proton scattering data from the self-consistent Granada-2013 database [28].

The Bochum-Bonn group has also been working on the chiral electromagnetic [29,30] and weak (axial) [31] current operators. First results with the $2 \mathrm{~N}$ electromagnetic currents from Ref. [29] were published in Refs. [32,33], but full-fledged calculations with the consistent Bochum-Bonn potentials and current operators will become possible, when the ongoing work on the regularization of the current operators is completed.

Concurrent with these studies have been the efforts by the Moscow(Idaho)-Salamanca group, which resulted in another family of nonlocal chiral NN potentials [34,35]. The most recent version of this potential, generated also up to fifth order in the chiral expansion, was published in Ref. [36]. At N4LO it reproduces the world $\mathrm{NN}$ data with the $\chi^{2} /$ datum of 1.08 for proton-proton and neutron-proton data up to $190 \mathrm{MeV}$.

Many modern calculations of various electromagnetic processes employ the chiral potentials from Refs. [34,35] and require chiral current operators. The latter were developed gradually, starting with a pioneering work by Park et al. [37]. The predictions of Refs. [37,38] were later rederived or supplemented by many authors [39-42], using various formulations of $\chi$ EFT. The unknown parameters of the effective theory were either related to the NN scattering or fixed by reproducing selected observables in the $2 \mathrm{~N}$ and $3 \mathrm{~N}$ systems, like the magnetic moments [43] and the tritium Gamow-Teller matrix element $[44,45]$. The derived current operators were used with the wave functions obtained with the traditional potentials and later, more consistently, with the potentials derived by the Moscow(Idaho)-Salamanca group. Among the many studied processes were those of direct astrophysical interest $[46,47]$, muon capture reactions [48-51] and, last but not least, neutrino-induced processes [52].

Predictions in Ref. [52] for inclusive neutrino scattering off the deuteron are fully based on a $\chi$ EFT input. The results concerning the cross sections are only slightly larger than the corresponding ones obtained in conventional formulations based on meson-exchange picture [3,8] and are insensitive to the value of the regulator parameter. This might indicate that the theoretical results have a very small uncertainty in the low-energy neutrino regime.

To give the reader a proper picture of the efforts aiming at the exact treatment of the neutrino-induced reactions, we mention here some calculations with heavier than $A=2$ nuclei. Gazit et al. performed a number of calculations for neutrino-induced break-up reactions with the ${ }^{3} \mathrm{H},{ }^{3} \mathrm{He}$, and ${ }^{4} \mathrm{He}$ nuclei [53-55], in which final-state interactions were included via the Lorentz integral transform method [56]. The resulting bound-state and bound-state-like equations were solved using the effective interaction hyperspherical harmonics approach [57,58], employing conventional $2 \mathrm{~N}$ and $3 \mathrm{~N}$ forces. While in Ref. [53] the impulse approximation was used, in Refs. [54,55] the nuclear current operator contained also $2 \mathrm{~N}$ contributions derived from $\chi$ EFT. Finally, we mention that weak inclusive responses of heavier light nuclei, including ${ }^{12} \mathrm{C}$, were investigated with the Green's function Monte Carlo method [59,60]. The results of these calculations contributed to the determination of the nucleon isovector axial form factor [61].

The momentum-space approach offers an independent possibility to perform calculations not only for the deuteron but also for the trinucleons' reactions with neutrinos. In the present work we calculate cross sections for several such reactions and build a solid base on which we can improve our dynamics in the future, adding many-nucleon forces and weak current operators. The present study, contrary to the very advanced investigations by Baroni et al. [52], is carried out with rather simple dynamical input. Namely we work with the traditional AV18 NN potential and restrict ourselves to the single-nucleon current. Thus we definitely cannot reach yet the high level of accuracy achieved by the predictions of Refs. [3,8,52], dealing solely with the neutrino-induced break-up of ${ }^{2} \mathrm{H}$. We agree with the statement in Ref. [52] that the accuracy of these predictions is very important in the analysis of the SNO experiments and more generally for our understanding of (anti)neutrino-nucleus scattering. Thus we decided to confront our momentum-space framework predictions with the above-mentioned results. We also agree with Ref. [52] that all these ingredients should be derived consistently from $\chi$ EFT. There are, however, still some open issues in the construction of the $2 \mathrm{~N}$ electroweak current operator, and the results for the axial current obtained by Krebs et al. [31] are not equivalent to those reported in Refs. [42,45]. Even if these differences prove to be of no practical importance, some fundamental questions about the consistence between chiral potentials and current operators still should be answered. Our framework is in any case ready for the improved input generated by $\chi$ EFT.

The results obtained within $\chi$ EFT are usually provided in momentum space and can be readily incorporated into momentum-space calculations. We refer the reader especially to the "three-dimensional" calculations, which avoid totally partial wave representation of nuclear states and operators $[62,63]$. In this approach $\chi$ EFT potentials and current operators would be used indeed directly, avoiding also convergence problems bound with partial wave decomposition. Our present results might thus provide a benchmark for such planned calculations.

Last but not least, momentum space framework allows one to systematically account for relativistic effects not only in the kinematics but also in the reaction dynamics. Some of such problems might be difficult to tackle in coordinate space but are easier to solve in momentum space. For example, the argument of the nucleon form factors in the single-nucleon current, which should be actually the four-momentum transfer to the nucleon squared, is usually replaced by the four-momentum transfer to the whole nuclear system squared. In momentum space one can directly use the proper values of the form factor arguments. 
Relativity plays definitely an important role for higher neutrino energies. Even at relatively low energies these effects have to be studied thoroughly, since approximate treatment of relativity adds to the total theoretical uncertainty of predictions. Ultimately, theoretical calculations should take into account complementary roles of kinematic and dynamical contributions to the Poincaré invariant formulation of reaction theory. Important examples of such investigations are given in Refs. [64-70]. A particular result of these studies, showing that relativistic effects in kinematics and dynamics might in fact partly cancel, prompted us to retain the nonrelativistic form of the phase-space factor, consistent with our nonrelativistic dynamics, in particular with the form of the current operator. Consequences of this choice will be discussed below.

The paper is organized in the following way. In Sec. II we introduce elements of our formalism and compare it with the calculations presented in Refs. [2,8]. In particular we discuss the differences in the treatment of kinematics and the current operator. In the following two sections we show selected results for various processes induced by neutrinos. Finally, Sec. V contains some concluding remarks and an outlook.

\section{ELEMENTS OF THE FORMALISM}

Recently, we have developed a framework to study several muon capture processes on the ${ }^{2} \mathrm{H},{ }^{3} \mathrm{H}$, and ${ }^{3} \mathrm{H}$ nuclei $[63,71]$. For the $\bar{v}_{l}+{ }^{2} \mathrm{H} \rightarrow l^{+}+n+n$ reaction, the transition from the initial to final state is also governed by the Fermi form of the interaction Lagrangian [72], again leading to a contraction of the leptonic $\left(\mathcal{L}_{\lambda}\right)$ and nuclear $\left(\mathcal{N}^{\lambda}\right)$ parts in the $S$ matrix element, $S_{f i}$ :

$$
S_{f i}=i(2 \pi)^{4} \delta^{4}\left(P^{\prime}-P\right) \frac{G_{F} \cos \theta_{C}}{\sqrt{2}} \mathcal{L}_{\lambda} \mathcal{N}^{\lambda},
$$

where the value of the Fermi constant, $G_{F}=1.1803 \times$ $10^{-5} \mathrm{GeV}^{-2}$, and $\cos \theta_{C}=0.97425$ have been deliberately taken to be the same as in Ref. [8]. The total initial (final) four-momentum is denoted as $P\left(P^{\prime}\right)$.

The leptonic matrix element

$$
\mathcal{L}_{\lambda}=\frac{1}{(2 \pi)^{3}} \bar{v}\left(\mathbf{k}, m_{\bar{\nu}}\right) \gamma_{\lambda}\left(1-\gamma_{5}\right) v\left(\mathbf{k}^{\prime}, m_{l^{+}}\right) \equiv \frac{1}{(2 \pi)^{3}} L_{\lambda}
$$

is given in terms of the Dirac spinors $v$ and the gamma matrices [73] and depends on the initial antineutrino three-momentum $\mathbf{k}$ and spin projection $m_{\bar{v}}$ as well as on the final antilepton three-momentum $\mathbf{k}^{\prime}$ and spin projection $m_{l^{+}}$. The same formula holds for the three lepton flavors $l=e, l=\mu$, and $l=\tau$.

The nuclear part

$$
\mathcal{N}^{\lambda}=\frac{1}{(2 \pi)^{3}}\left\langle\Psi_{f} \mathbf{P}_{f} m_{f}\left|j_{\mathrm{CC}}^{\lambda}\right| \Psi_{i} \mathbf{P}_{i} m_{i}\right\rangle \equiv \frac{1}{(2 \pi)^{3}} N_{\mathrm{CC}}^{\lambda}
$$

is a matrix element of the nuclear weak charged current (CC) operator $j_{\mathrm{CC}}^{\lambda}$ between the initial and final nuclear states. The total initial (final) nuclear three-momentum is denoted as $\mathbf{P}_{i}$ $\left(\mathbf{P}_{f}\right), m_{i}$ is the initial nucleus spin projection, and $m_{f}$ is the set of spin projections in the final state. In this paper we restrict ourselves to the single-nucleon current operator with relativistic corrections. This current operator was defined in Eqs. (2.9) and (2.10) of Ref. [63]. It is very close to the one used in Ref. [49] and employs form factors, whose explicit expressions and parametrization can be found in Ref. [8]. Thus, except for one term which is numerically insignificant, we use the same single-nucleon current operator as in Ref. [63].

On top of the single-nucleon operators, also many-nucleon contributions appear in $j_{\mathrm{CC}}^{\lambda}$. Their role has been studied, for example, in Ref. [49]. For the neutrino-induced reactions of interest, the effects of $2 \mathrm{~N}$ contributions in the weak current operator were estimated in Ref. [8] to be smaller than $10 \%$ over the wide energy range from the threshold to $\mathrm{GeV}$ energies. Thus we decided to base our first predictions on the singlenucleon current only and represent all dynamical ingredients in momentum space.

The only change in the charged single-nucleon current operator for the $v_{l}+{ }^{2} \mathrm{H} \rightarrow l^{-}+p+p$ process compared to the $\bar{v}_{l}+{ }^{2} \mathrm{H} \rightarrow l^{+}+n+n$ reaction is the replacement of the overall isospin lowering operator by the isospin raising operator:

$$
\tau_{-} \equiv\left(\tau_{x}-\mathrm{i} \tau_{y}\right) / 2 \longrightarrow \tau_{+} \equiv\left(\tau_{x}+\mathrm{i} \tau_{y}\right) / 2 .
$$

However, since the matrix elements of the single-nucleon operator in the $2 \mathrm{~N}$ isospin space, spanned by the $\left|\left(\frac{1}{2} \frac{1}{2}\right) t m_{t}\right\rangle$ states,

$$
\left\langle\left(\frac{1}{2} \frac{1}{2}\right) 1-1\left|\tau_{-}(1)\right|\left(\frac{1}{2} \frac{1}{2}\right) 00\right\rangle
$$

and

$$
\left\langle\left(\frac{1}{2} \frac{1}{2}\right) 11\left|\tau_{+}(1)\right|\left(\frac{1}{2} \frac{1}{2}\right) 00\right\rangle,
$$

have just an opposite sign, we can use for this reaction exactly the same single-nucleon current operator. Its matrix elements, $N_{\mathrm{CC}}^{\lambda}$, are contracted with the altered leptonic matrix elements

$$
L_{\lambda}=\bar{u}\left(\mathbf{k}^{\prime}, m_{l^{-}}\right) \gamma_{\lambda}\left(1-\gamma_{5}\right) u\left(\mathbf{k}, m_{\nu}\right),
$$

expressed through the Dirac spinors $u$, which depend on the initial neutrino three-momentum $\mathbf{k}$ and spin projection $m_{v}$ as well as on the final lepton three-momentum $\mathbf{k}^{\prime}$ and spin projection $m_{l^{-}}$. In the following, the energy of the initial (anti)neutrino will be denoted by $E$ and for the massless (anti)neutrino $E=|\mathbf{k}|$.

\section{A. Kinematics}

Since we compare our nonrelativistic predictions with the ones published in Ref. [8], where the relativistic kinematics was employed, we give here some formulas for our kinematics and cross sections. We believe that they will be useful in the future benchmark calculations and serve to disentangle relativistic kinematical effects from dynamical ones. The difference in the treatment of kinematics is the main reason our predictions diverge from the results published in Ref. [8], especially for higher energies.

The kinematics of the $\bar{v}_{l}+{ }^{2} \mathrm{H} \rightarrow l^{+}+n+n$ and $v_{l}+$ ${ }^{2} \mathrm{H} \rightarrow l^{-}+p+p$ processes is essentially identical (the only difference being the mass of two identical nucleons in the final state) and can be treated both relativistically and nonrelativistically. The relativistic formulas for different kinematical 
TABLE I. Threshold energies in MeV for various (anti)neutrinoinduced reactions on the deuteron calculated relativistically $\left(E_{\mathrm{thr}}^{\mathrm{rel}}\right)$ and nonrelativistically $\left(E_{\mathrm{thr}}^{\mathrm{nrl}}\right)$. We assumed $M_{d}=1875.613 \mathrm{MeV}, M_{p}=$ 938.272 MeV, $M_{n}=939.565 \mathrm{MeV}$, and $M_{e}=0.510999 \mathrm{MeV}$. Results for the $v_{l}+d \rightarrow v_{l}+p+n$ and $\bar{v}_{l}+d \rightarrow \bar{v}_{l}+p+n$ processes are identical for all the flavors $l=e, \mu, \tau$.

\begin{tabular}{lcc}
\hline \hline Reaction & $E_{\mathrm{thr}}^{\mathrm{rel}}$ & $E_{\mathrm{thr}}^{\mathrm{nrl}}$ \\
\hline $\bar{\nu}_{e}+d \rightarrow e^{+}+n+n$ & 4.03323 & 4.03323 \\
$\nu_{e}+d \rightarrow e^{-}+p+p$ & 1.44279 & 1.44279 \\
$\nu_{l}+d \rightarrow v_{l}+p+n$ & 2.22589 & 2.22589 \\
\hline \hline
\end{tabular}

quantities are given in Refs. [2,8], so we can focus on the differences between the exact relativistic and our approximate nonrelativistic treatment of nuclear kinematics. (Note that lepton kinematics is always treated relativistically.) The starting point is the energy and momentum conservation, where we neglect the very small (anti)neutrino mass and assume that the initial deuteron is at rest. In the nonrelativistic formulation for the nuclear sector it reads:

$$
\begin{aligned}
E+M_{d} & =\sqrt{M_{l}^{2}+\mathbf{k}^{\prime 2}}+2 M_{p}+\frac{\mathbf{p}_{1}^{2}}{2 M_{p}}+\frac{\mathbf{p}_{2}^{2}}{2 M_{p}}, \\
\mathbf{k} & =\mathbf{k}^{\prime}+\mathbf{p}_{1}+\mathbf{p}_{2},
\end{aligned}
$$

where $\mathbf{p}_{1}$ and $\mathbf{p}_{2}$ stand for the individual momenta of the two outgoing nucleons. The deuteron, nucleon, and (anti)lepton masses are denoted as $M_{d}, M_{p}$, and $M_{l}$, respectively. Starting from Eqs. (2.6) all the necessary kinematical quantities can be calculated.

We begin with the (anti)neutrino threshold energy, $E_{\mathrm{thr}}$, rewriting Eq. (2.6) as

$$
E+M_{d}=\sqrt{M_{l}^{2}+\mathbf{k}^{\prime 2}}+2 M_{p}+\frac{\mathbf{p}_{12}^{2}}{4 M_{p}}+\frac{\mathbf{p}^{2}}{M_{p}},
$$

where $\mathbf{p}_{12} \equiv \mathbf{p}_{1}+\mathbf{p}_{2}=\mathbf{k}-\mathbf{k}^{\prime}$ and $\mathbf{p}=\frac{1}{2}\left(\mathbf{p}_{1}-\mathbf{p}_{2}\right)$. Next we numerically seek the smallest possible value of $E$ (represented by $E_{\mathrm{thr}}^{\mathrm{nrl}}$ ), for which a physical solution of

$$
E+M_{d}=\sqrt{M_{l}^{2}+\mathbf{k}^{\prime 2}}+2 M_{p}+\left(\mathbf{k}-\mathbf{k}^{\prime}\right)^{2} 4 M_{p}
$$

exists. Obviously the same logic applies to the $v_{l}+d \rightarrow$ $v_{l}+p+n$ and $\bar{v}_{l}+d \rightarrow \bar{v}_{l}+p+n$ reactions. In this case we additionally neglect the small difference between the proton mass $M_{p}$ and neutron mass $M_{n}$ and use the average "nucleon mass," $M \equiv \frac{1}{2}\left(M_{p}+M_{n}\right)$. From Table I, where we display the numerical results for the threshold energies, it is clear that differences between the relativistic and nonrelativistic results are insignificant.

We determine also the maximal energy of the emerging lepton under a given scattering angle $\theta$, where $\cos \theta=\hat{\mathbf{k}} \cdot \hat{\mathbf{k}}^{\prime}$. Note that there is no restriction on the scattering angle $\theta$. Nonrelativistically, the kinetic energy of the $2 \mathrm{~N}$ system in the $2 \mathrm{~N}$ total momentum zero frame must be non-negative:

$$
E+M_{d}-2 M_{p}-\sqrt{M_{l}^{2}+\mathbf{k}^{\prime 2}}-\frac{\left(\mathbf{k}-\mathbf{k}^{\prime}\right)^{2}}{4 M_{p}} \geqslant 0 .
$$

This condition yields now a fourth-degree equation:

$$
\begin{aligned}
& k^{\prime 4}-4 E \cos \theta k^{\prime 3} \\
& +\left(4 E^{2} \cos ^{2} \theta-16 M_{p}^{2}-2 W\right) k^{\prime 2} \\
& +4 W E \cos \theta k^{\prime}+W^{2}-16 M_{p}^{2} M_{l}^{2}=0,
\end{aligned}
$$

with $W \equiv 4 M_{p}\left(E+M_{d}-2 M_{p}\right)-E^{2}$. One of the roots of Eq. (2.10) represents the maximal value of the magnitude of the outgoing lepton momentum, $\left(k^{\prime}\right)_{\max }^{\mathrm{nrl}}$. We compared $\left(k^{\prime}\right)_{\max }^{\mathrm{nrl}}$ with its relativistic analogue $\left(k^{\prime}\right)_{\max }^{\mathrm{rel}}$ and found that even at $E=300 \mathrm{MeV}$ the maximal relative difference between these values, noticed for the backward angles, did not reach $0.5 \%$.

Finally, we want to discuss the phase-space factor. In Refs. [2,8] two different relativistic forms are employed. With $\omega \equiv E-\sqrt{M_{l}^{2}+\mathbf{k}^{\prime 2}} \equiv E-E^{\prime}$ and $\mathbf{Q} \equiv \mathbf{k}-\mathbf{k}^{\prime}$ the nonrelativistic evaluation starts from

$$
\begin{aligned}
\frac{d \sigma}{d^{3} \mathbf{k}^{\prime}} \sim & \delta\left(\omega+M_{d}-2 M_{p}-\frac{\mathbf{p}_{1}^{2}}{2 M_{p}}-\frac{\mathbf{p}_{2}^{2}}{2 M_{p}}\right) \\
& \times \delta^{3}\left(\mathbf{Q}-\mathbf{p}_{1}-\mathbf{p}_{2}\right) d^{3} \mathbf{p}_{1} d^{3} \mathbf{p}_{2}
\end{aligned}
$$

and the change of variables, $\mathbf{p}_{12}=\mathbf{p}_{1}+\mathbf{p}_{2}, \mathbf{p}=\left(\mathbf{p}_{1}-\mathbf{p}_{2}\right)$, yields after standard steps

$$
\frac{d \sigma}{d^{3} \mathbf{k}^{\prime}} \sim \int_{-1}^{1} d x \frac{1}{2} M_{p} p_{\mathrm{nrl}}
$$

where $p_{\mathrm{nrl}}=\sqrt{M_{p}\left(\omega+M_{d}-2 M_{p}-\frac{Q^{2}}{4 M_{p}}\right)}$ and we defined $x \equiv \hat{\mathbf{p}} \cdot \hat{\mathbf{Q}}$ and $Q \equiv|\mathbf{Q}|$.

In Fig. 1 we compare the relativistic phase-space factor from Ref. [8], $\rho_{\text {rel }}=\frac{p^{2}}{\left|\frac{p+Q x / 2}{E_{1}}+\frac{p-Q x / 2}{E_{2}}\right|}$, and our nonrelativistic one, $\rho_{\mathrm{nrl}}=\frac{1}{2} M_{p} p_{\mathrm{nrl}}$. For a fixed initial electron neutrino energy and just one lepton scattering angle $\theta=\pi / 2$, we calculate the phase-space factors as a function of the outgoing lepton momentum $k^{\prime}$. The relativistic phase-space factor depends not only on the magnitude of the relative momentum $p$ but also on $x$, so we calculate $\rho_{\text {rel }}$ for seven $x$ values, $-1,-2 / 3,-1 / 3$, $0,1 / 3,2 / 3$, and 1 , to check how strong the dependence on $x$ is. For $E=50 \mathrm{MeV}$ all upper curves representing relativistic results with different $x$ values essentially overlap, but for $E=300 \mathrm{MeV}$ the spread due to the different $x$ values is clearly visible. The relativistic phase-space factors are larger in the whole range of the $k^{\prime}$ momentum. The difference is particularly strong for small $k^{\prime}$ values and for $E=300 \mathrm{MeV}$ it exceeds $20 \%$. For $E=50 \mathrm{MeV}$ it is much smaller and reaches about $3 \%$. As already mentioned, the difference in the phase-space factor is the main reason why our predictions for the total cross sections for (anti)neutrino scattering on ${ }^{2} \mathrm{H}$ diverge from the results presented in Ref. [8].

\section{B. The $2 \mathrm{~N}$ scattering states in coordinate and momentum spaces}

The nuclear matrix element, $\left\langle\Psi_{f} \mathbf{P}_{f} m_{f}\left|j_{\mathrm{CC}}^{\lambda}\right| \Psi_{i} \mathbf{P}_{i} m_{i}\right\rangle$, involves the initial deuteron state $\left|\phi_{d} m_{d}\right\rangle$ and the $2 \mathrm{~N}$ scattering state $\left|\mathbf{p} m_{1} m_{2}\right\rangle^{(-)}$, where the latter is formally defined as

$$
\left|\mathbf{p} m_{1} m_{2}\right\rangle^{(-)} \equiv \lim _{\epsilon \rightarrow 0^{+}} \frac{-i \epsilon}{E_{2 \mathrm{~N}}-i \epsilon-H_{0}-V}\left|\mathbf{p} m_{1} m_{2}\right\rangle
$$



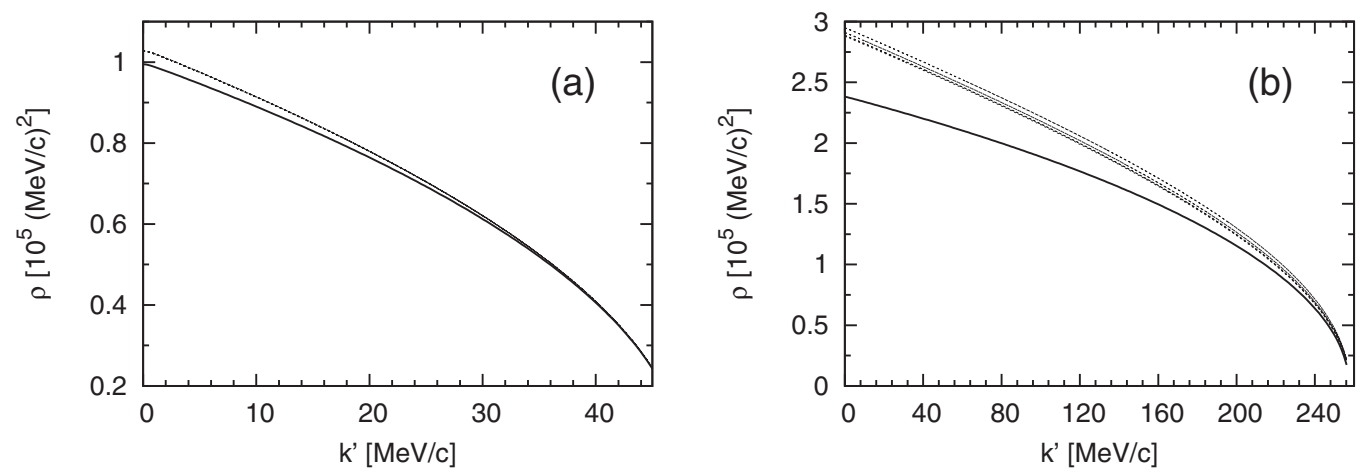

FIG. 1. The relativistic (group of dotted lines) and nonrelativistic (solid line) phase-space factors compared for the initial neutrino energy $E=50 \mathrm{MeV}$ (a) and $E=300 \mathrm{MeV}$ (b) for the lepton scattering angle $\theta=\pi / 2$ as a function of the outgoing lepton momentum $k^{\prime}$. The group of dotted curves represent results with different values of $x$ (see text). Note that these different predictions overlap for $E=50 \mathrm{MeV}$.

and in practice is computed from the scattering $t$ matrix as

$$
\begin{aligned}
& \left|\mathbf{p} m_{1} m_{2}\right\rangle^{(-)} \\
& \quad=\lim _{\epsilon \rightarrow 0^{+}}\left[1+G_{0}^{2 \mathrm{~N}}\left(E_{2 \mathrm{~N}}-i \epsilon\right) t\left(E_{2 \mathrm{~N}}-i \epsilon\right)\right]\left|\mathbf{p} m_{1} m_{2}\right\rangle,
\end{aligned}
$$

where $G_{0}^{2 \mathrm{~N}}\left(E_{2 \mathrm{~N}}\right)$ is the free $2 \mathrm{~N}$ propagator and the kinetic energy of the relative motion in the $2 \mathrm{~N}$ system in our nonrelativistic approximation, $E_{2 \mathrm{~N}}=\frac{\mathbf{p}^{2}}{M}$, is given by Eq. (2.7). The $t$ matrix is obtained, for a given $\mathrm{NN}$ potential $V$, as a solution of the Lippmann-Schwinger equation:

$$
t\left(E_{2 \mathrm{~N}}\right)=V+t\left(E_{2 \mathrm{~N}}\right) G_{0}^{2 \mathrm{~N}}\left(E_{2 \mathrm{~N}}\right) V .
$$

Using Eq. (2.14), the nuclear matrix element, $N^{\lambda} \equiv$ $\left\langle\Psi_{f} \mathbf{P}_{f} m_{f}\left|j_{\mathrm{CC}}^{\lambda}\right| \Psi_{i} \mathbf{P}_{i} m_{i}\right\rangle$, becomes

$$
\begin{aligned}
N^{\lambda}= & \left\langle\mathbf{p} \mathbf{P}_{f}=\mathbf{k}-\mathbf{k}^{\prime} m_{1} m_{2}\right|\left[1+t\left(E_{2 \mathrm{~N}}\right) G_{0}^{2 \mathrm{~N}}\left(E_{2 \mathrm{~N}}\right)\right] \\
& \times j_{\mathrm{CC}}^{\lambda}\left|\phi_{d} \mathbf{P}_{i}=0 m_{d}\right\rangle \\
= & \left\langle\mathbf{p} m_{1} m_{2}\right|\left[1+t\left(E_{2 \mathrm{~N}}\right) G_{0}^{2 \mathrm{~N}}\left(E_{2 \mathrm{~N}}\right)\right] \\
& \times j_{\mathrm{CC}}^{\lambda}\left(\mathbf{P}_{f}, \mathbf{P}_{i}\right)\left|\phi_{d} m_{d}\right\rangle .
\end{aligned}
$$

As already stated, we generate the deuteron wave function and solve Eq. (2.15) in momentum space, using the $2 \mathrm{~N}$ partial wave states, $\left|p(l s) j m_{j} ; t m_{t}\right\rangle$. They carry information about the magnitude of the relative momentum $(p)$, the relative angular momentum $(l)$, spin $(s)$, and total angular momentum $(j)$ with its corresponding projection $\left(m_{j}\right)$. This set of quantum numbers is supplemented by the $2 \mathrm{~N}$ isospin $(t)$ and its projection $\left(m_{t}\right)$. In the present work we employ all partial wave states with $j \leqslant 4$. Such calculations, closely corresponding to the ones presented in Ref. [63], are fully sufficient for the antineutrinoinduced $\mathrm{CC}$ reactions and the NC-driven reactions, where only short-range potentials act between the two outgoing nucleons.

The neutrino-induced CC reactions lead, however, in the final state to two protons, which interact also by the long-range Coulomb potential. The $2 \mathrm{~N}$ scattering problem involving this interaction is usually solved in coordinate space. We could follow the steps outlined in Ref. [8], but we wanted to take advantage of momentum space framework developed for the muon capture reaction. That is why we decided to perform standard momentum space $t$ matrix calculations for the short- range potential. Thus the proton-proton version of the AV18 potential was supplemented by the restricted Coulomb (RC) sharply cut off force $V_{\mathrm{RC}}$, whose matrix elements are given by the following integral:

$$
\begin{aligned}
& \left\langle p^{\prime}\left(l^{\prime} s^{\prime}\right) j^{\prime} m_{j^{\prime}} ; t^{\prime} m_{t^{\prime}}\left|V_{\mathrm{RC}}\right| p(l s) j m_{j} ; t m_{t}\right\rangle \\
& =\delta_{l l^{\prime}} \delta_{s s^{\prime}} \delta_{j j^{\prime}} \delta_{m_{j} m_{j^{\prime}}} \delta_{t t^{\prime}} \delta_{m_{t} m_{t^{\prime}}} \delta_{t 1} \delta_{m_{t} 1} 8 \alpha \\
& \quad \times \int_{0}^{R_{C}} d r r j_{l}\left(p^{\prime} r\right) j_{l}(p r),
\end{aligned}
$$

where $j_{l}(p r)$ is the spherical Bessel function and $\alpha$ is the fine structure constant. The value of the sharp cut-off was taken to be $R_{C}=40 \mathrm{fm}$. This approach is fully justified by the observation that the current matrix elements Bessel transformed to coordinate space,

$$
\begin{aligned}
& \left\langle r(l s) j m_{j}\left|j_{\mathrm{CC}}^{\lambda}\right| \phi_{d} m_{d}\right\rangle \\
& \quad=\frac{2}{\pi} i^{l} \int_{0}^{\infty} d k k^{2} j_{l}(k r)\left\langle k(l s) j m_{j}\left|j_{\mathrm{CC}}^{\lambda}\right| \phi_{d} m_{d}\right\rangle,
\end{aligned}
$$

become negligible for $r \geqslant 30 \mathrm{fm}$.

Additionally, we checked that our momentum space generated $2 \mathrm{~N}$ scattering states are fully equivalent to the radial wave functions calculated directly in coordinate space, using the collocation method from Refs. [74-76]. To this end we employed the well-known formula (see, for example, Ref. [8] and references therein), which using our normalization of states, reads

$$
\begin{aligned}
\psi_{l^{\prime} s l s}^{j}(r)= & \delta_{l^{\prime} l} j_{l}(p r)+i^{l-l^{\prime}} M \\
& \times \int_{0}^{\infty} \frac{d k k^{2} j_{l^{\prime}}(k r)}{p^{2}-k^{2}+i \epsilon}\left\langle k\left(l^{\prime} s\right) j\left|t\left(E_{2 \mathrm{~N}}\right)\right| p(l s) j\right\rangle .
\end{aligned}
$$

\section{The cross section}

Having discussed all the elements of our formalism, we can give the main formula for the cross section, consistent with the momentum space formalism presented in the previous subsections. We do it for the $v_{e}+{ }^{2} \mathrm{H} \rightarrow e^{-}+p+p$ reaction and discuss later some differences if other reactions are 
considered. With our normalization of the $N^{\lambda}$ matrix elements we start with [73]

$$
\begin{aligned}
d \sigma= & \frac{1}{\left|\mathbf{v}_{1}-\mathbf{v}_{2}\right|} \frac{1}{2 E}\left(L_{\alpha}\right)^{*} L_{\beta}\left(N^{\alpha}\right)^{*} N^{\beta} \frac{G_{F}^{2} \cos ^{2} \theta_{C}}{2} \\
& \times F\left(Z, k^{\prime}\right) \frac{d^{3} \mathbf{k}^{\prime}}{2 E^{\prime}} \frac{d^{3} \mathbf{p}_{1}}{(2 \pi)^{3}} \frac{d^{3} \mathbf{p}_{2}}{(2 \pi)^{3}}(2 \pi)^{4} \delta^{4}\left(P^{\prime}-P\right) \mathcal{S}
\end{aligned}
$$

where in the laboratory frame the relative velocity of the projectile and target $\left|\mathbf{v}_{1}-\mathbf{v}_{2}\right|$ is equal $c(=1)$ and the $\mathcal{S}$ factor is needed when in the final-state identical particles appear. The Fermi function $F\left(Z, k^{\prime}\right)$ [77] is introduced to account for the Coulomb modification of the final lepton wave functions by the two protons in the final state and is not needed otherwise. (Note that in $L_{\alpha}$ we use the following normalization for the Dirac spinors: $\bar{u} u=2 M_{l}$ and $\bar{v} v=-2 M_{l}$.) Defining

$$
\widetilde{L_{\alpha \beta}}=\sum_{m_{v}} \sum_{m_{l^{-}}}\left(L_{\alpha}\right)^{*} L_{\beta},
$$

taking all factors into account and evaluating the phase-space factor in terms of the relative momentum, we arrive at the following expression for the total cross section:

$$
\begin{aligned}
\sigma_{\mathrm{tot}}= & \frac{G_{F}^{2} \cos ^{2} \theta_{C}}{2(2 \pi)^{2}} \frac{1}{4 E} \int_{0}^{2 \pi} d \phi \int_{0}^{\pi} d \theta \sin \theta \\
& \int_{M_{l}}^{E_{\text {max }}^{\prime}} d E^{\prime} k^{\prime} \frac{1}{2} M_{p} p_{\mathrm{nrl}} F\left(Z, k^{\prime}\right) \\
& \times \int_{0}^{2 \pi} d \phi_{p} \int_{0}^{\pi} d \theta_{p} \sin \theta_{p} \frac{2}{3} \sum_{m_{1}, m_{2}} \sum_{m_{d}} \widetilde{L_{\alpha \beta}}\left(N^{\alpha}\right)^{*} N^{\beta},
\end{aligned}
$$

where the $\theta$ and $\phi$ angles describe the direction of the outgoing lepton in the laboratory frame. Note that for the total unpolarized cross section considered here the integral over the azimuthal angle $\phi$ can be replaced by the factor $2 \pi$.

The contraction $|T|^{2} \equiv \widetilde{L_{\alpha \beta}}\left(N^{\alpha}\right)^{*} N^{\beta}$ can be written in terms of the $V_{i j}$ functions stemming from the lepton arm and the products of the nuclear matrix elements $N^{\alpha}$. For the latter we use the spherical components and obtain

$$
\begin{aligned}
|T|^{2}= & V_{00}\left|N^{0}\right|^{2}+V_{M M}\left|N_{-1}\right|^{2}+V_{P P}\left|N_{+1}\right|^{2} \\
& +V_{Z Z}\left|N_{z}\right|^{2}+V_{Z 0} N_{z}\left(N^{0}\right)^{*}+V_{0 Z} N^{0}\left(N_{z}\right)^{*},
\end{aligned}
$$

where for the neutrino-induced reactions

$$
\begin{aligned}
V_{00} & =8\left(\mathbf{k}^{\prime} \cdot \mathbf{k}+E E^{\prime}\right), \\
V_{M M} & =8\left(E+k_{z}\right)\left(E^{\prime}-k_{z}^{\prime}\right), \\
V_{P P} & =8\left(E-k_{z}\right)\left(E^{\prime}+k_{z}^{\prime}\right), \\
V_{Z Z} & =8\left(-\mathbf{k}^{\prime} \cdot \mathbf{k}+E E^{\prime}\right), \\
V_{Z 0} & =-8\left(E k_{z}^{\prime}+E^{\prime} k_{z}\right)+8 i\left(k_{y}^{\prime} k_{x}-k_{x}^{\prime} k_{y}\right), \\
V_{0 Z} & =-8\left(E k_{z}^{\prime}+E^{\prime} k_{z}\right)+8 i\left(k_{x}^{\prime} k_{y}-k_{y}^{\prime} k_{x}\right) .
\end{aligned}
$$

The corresponding $\bar{V}_{i j}$ functions for the antineutrinoinduced reactions are given as:

$$
\begin{aligned}
\bar{V}_{00} & =V_{00}, \\
\bar{V}_{M M} & =V_{P P}, \\
\bar{V}_{P P} & =V_{M M}, \\
\bar{V}_{Z Z} & =V_{Z Z}, \\
\bar{V}_{Z 0} & =V_{0 Z}, \\
\bar{V}_{0 Z} & =V_{Z 0} .
\end{aligned}
$$

In the following we assume the system of coordinates, where $\mathbf{Q} \equiv \mathbf{k}-\mathbf{k}^{\prime} \| \hat{z}$ and $\hat{y}=\frac{\mathbf{k} \times \mathbf{k}^{\prime}}{\left|\mathbf{k} \times \mathbf{k}^{\prime}\right|}$, so

$$
\begin{aligned}
k_{x}^{\prime} & =k_{x}=|\mathbf{k}|\left|\mathbf{k}^{\prime}\right| \sin \theta /|\mathbf{Q}|, \\
k_{y}^{\prime} & =k_{y}=0, \\
k_{z} & =|\mathbf{k}|\left(|\mathbf{k}|-\left|\mathbf{k}^{\prime}\right| \cos \theta\right) /|\mathbf{Q}|, \\
k_{z}^{\prime} & =\left|\mathbf{k}^{\prime}\right|\left(-\left|\mathbf{k}^{\prime}\right|+|\mathbf{k}| \cos \theta\right) /|\mathbf{Q}|, \\
|\mathbf{Q}| & =\sqrt{\mathbf{k}^{2}+\mathbf{k}^{\prime 2}-2|\mathbf{k}|\left|\mathbf{k}^{\prime}\right| \cos \theta} .
\end{aligned}
$$

As a consequence, we get further simplifications:

$$
\bar{V}_{0 Z}=\bar{V}_{Z 0}=V_{0 Z}=V_{Z 0} \text {. }
$$

The main reason to use the spherical components of the current operator and the system of coordinates defined above is that we get in this case the simplest relations between the total spin magnetic quantum numbers $m_{d}$ and $m_{j}$ for matrix elements $\left\langle p(l s) j m_{j}\left|j_{\mathrm{CC}}^{\lambda}\right| \phi_{d} m_{d}\right\rangle$ in the partial wave representation:

$$
\begin{aligned}
& \left\langle p(l s) j m_{j}\left|j_{\mathrm{CC}}^{0}\right| \phi_{d} m_{d}\right\rangle \propto \delta_{m_{j}, m_{d}}, \\
& \left\langle p(l s) j m_{j}\left|j_{C C, z}\right| \phi_{d} m_{d}\right\rangle \propto \delta_{m_{j}, m_{d}}, \\
& \left\langle p(l s) j m_{j}\left|j_{C C, \pm 1}\right| \phi_{d} m_{d}\right\rangle \propto \delta_{m_{j} \pm 1, m_{d}} .
\end{aligned}
$$

For the NC-driven processes Eq. (2.22) has to be modified. The Fermi function $F\left(Z, k^{\prime}\right)$ and $\cos ^{2} \theta_{C}$ are replaced by 1 , but most importantly the weak CC operator $j_{\mathrm{CC}}^{\lambda}$ is replaced by the corresponding NC operator $j_{\mathrm{NC}}^{\lambda}$. Its construction is described in detail in Refs. [2,8] and we follow Ref. [8] for the choice of the nucleon form factors. Since we employ only the singlenucleon current, we use the given prescription for the proton and neutron NC operators and, using the isospin formalism, define the current of nucleon $i$ as

$$
j_{\mathrm{NC}}(i)=\frac{1}{2}\left(1+\tau_{3}(i)\right) j_{\mathrm{NC}}^{p}+\frac{1}{2}\left(1-\tau_{3}(i)\right) j_{\mathrm{NC}}^{n}
$$

in full analogy to the electromagnetic single-nucleon current. Of course, also in this case the relations (2.28) remain true.

\section{The $3 N$ matrix elements}

We treat the ${ }^{3} \mathrm{He}\left({ }^{3} \mathrm{H}\right)$ disintegration process analogously to the $2 \mathrm{~N}$ reactions. The $3 \mathrm{~N}$ Hamiltonian $H$ comprises the $3 \mathrm{~N}$ kinetic energy $\left(H_{0}\right)$, two-body subsystem potential energies $\left(V_{12}, V_{23}\right.$, and $\left.V_{31}\right)$, as well as the three-body potential energy $\left(V_{123}\right)$. The latter quantity is usually called a $3 \mathrm{~N}$ force $(3 \mathrm{NF})$ and is decomposed into three terms,

$$
V_{123}=V^{(1)}+V^{(2)}+V^{(3)}
$$


where $V^{(i)}$ is symmetric under exchange of nucleons $j$ and $k(i, j, k=1,2,3, i \neq j \neq k \neq i)$. The $3 \mathrm{~N}$ bound-state wave function is calculated using the method described in Ref. [78]. The Faddeev equation for the Faddeev component $|\psi\rangle$ reads

$$
|\psi\rangle=G_{0} t_{23} P|\psi\rangle+\left(1+G_{0} t_{23}\right) G_{0} V^{(1)}(1+P)|\psi\rangle .
$$

Here $G_{0} \equiv 1 /\left(E-H_{0}\right)$ is the free $3 \mathrm{~N}$ propagator and $P \equiv$ $P_{12} P_{23}+P_{13} P_{23}$ is the permutation operator built from transpositions $P_{i j}$, which interchange nucleons $i$ and $j$. Note that the two-body $t$ operator $t_{23}$ acts now in the $3 \mathrm{~N}$ space. The full wave function $|\Psi\rangle$ is easily obtained from the Faddeev component as

$$
|\Psi\rangle=(1+P)|\psi\rangle .
$$

The $3 \mathrm{~N}$ current operator $j_{3 \mathrm{~N}}^{\mu}$ contains the single-nucleon, $2 \mathrm{~N}$ and, in principle, also the $3 \mathrm{~N}$ contribution. Therefore we write:

$$
j_{3 \mathrm{~N}}^{\mu}=j_{1}^{\mu}+j_{2}^{\mu}+j_{3}^{\mu}+j_{12}^{\mu}+j_{23}^{\mu}+j_{31}^{\mu}+j_{123}^{\mu},
$$

where the $3 \mathrm{~N}$ part can be split into three components (just like the $3 \mathrm{NF}), j_{123}^{\mu}=j^{\mu 1}+j^{\mu 2}+j^{\mu 3}$. Thus we can decompose the $3 \mathrm{~N}$ current operator into three parts, $j^{\mu}(i)(i=1,2,3)$, which possess the same symmetry properties as $V^{(i)}$ :

$$
j_{3 \mathrm{~N}}^{\mu}=j^{\mu}(1)+j^{\mu}(2)+j^{\mu}(3),
$$

where, for example, $j^{\mu}(1) \equiv j_{1}^{\mu}+j_{23}^{\mu}+j^{\mu 1}$.

With all these ingredients, we construct the matrix elements for the nucleon-deuteron $(\mathrm{Nd})$

$$
N_{\mathrm{Nd}}^{\mu}=\left\langle\Psi_{\mathrm{Nd}}^{(-)}\left|j_{3 \mathrm{~N}}^{\mu}\right| \Psi\right\rangle
$$

and the $3 \mathrm{~N}$ break-up channel

$$
N_{3 \mathrm{~N}}^{\mu}=\left\langle\Psi_{3 \mathrm{~N}}^{(-)}\left|j_{3 \mathrm{~N}}^{\mu}\right| \Psi\right\rangle,
$$

with the corresponding channel scattering states. To this end first we solve a Faddeev-type equation for an auxiliary state $\left|U^{\mu}\right\rangle[79]$,

$$
\begin{aligned}
\left|U^{\mu}\right\rangle= & {\left[t_{23} G_{0}+\frac{1}{2}(1+P) V^{(1)} G_{0}\left(1+t_{23} G_{0}\right)\right] } \\
& \times(1+P) j^{\mu}(1)|\Psi\rangle \\
& +\left[t_{23} G_{0} P+\frac{1}{2}(1+P) V^{(1)} G_{0}\left(1+t_{23} G_{0}\right) P\right]\left|U^{\mu}\right\rangle,
\end{aligned}
$$

which depends on the component of the current operator and two kinematical quantities but is independent of the final-state kinematics. The two kinematical quantities are the $3 \mathrm{~N}$ internal, center of mass (c.m.), energy $E_{\mathrm{c} . \mathrm{m} .}$ and the magnitude of the three-momentum transferred to the $3 \mathrm{~N}$ system, $|\mathbf{Q}|$. In practice we use the density operator $\rho \equiv j_{3 \mathrm{~N}}^{0}$ as well as the spherical components of the current operator

$$
\begin{aligned}
& j_{+1} \equiv-\frac{1}{\sqrt{2}}\left(j_{3 \mathrm{~N}}^{1}+i j_{3 \mathrm{~N}}^{2}\right) \equiv-\frac{1}{\sqrt{2}}\left(j_{x 3 N}+i j_{y 3 N}\right), \\
& j_{-1} \equiv \frac{1}{\sqrt{2}}\left(j_{3 \mathrm{~N}}^{1}-i j_{3 \mathrm{~N}}^{2}\right) \equiv \frac{1}{\sqrt{2}}\left(j_{x 3 N}-i j_{y 3 N}\right),
\end{aligned}
$$

and choose $\mathbf{Q}$ parallel to the $z$ axis. As in the $2 \mathrm{~N}$ case, this yields the simplest relations between the total $3 \mathrm{~N}$ angular momentum projections of the initial and final nuclear systems.
The matrix elements $N_{\mathrm{Nd}}^{\mu}$ and $N_{3 \mathrm{~N}}^{\mu}$ for arbitrary exclusive kinematics are then obtained by simple quadratures:

$N_{\mathrm{Nd}}^{\mu}=\left\langle\phi_{\mathrm{Nd}}\left|(1+P) j^{\mu}(1)\right| \Psi\right\rangle+\left\langle\phi_{\mathrm{Nd}}|P| U^{\mu}\right\rangle$,

$N_{3 \mathrm{~N}}^{\mu}=\left\langle\phi_{3 \mathrm{~N}}\left|(1+P) j^{\mu}(1)\right| \Psi\right\rangle+\left\langle\phi_{3 \mathrm{~N}}\left|t_{23} G_{0}(1+P) j^{\mu}(1)\right| \Psi\right\rangle$

$$
+\left\langle\phi_{3 \mathrm{~N}}|P| U^{\mu}\right\rangle+\left\langle\phi_{3 \mathrm{~N}}\left|t_{23} G_{0} P\right| U^{\mu}\right\rangle,
$$

where $\left|\phi_{\mathrm{Nd}}\right\rangle$ is a product of the internal deuteron state and the state describing the free relative motion of the third nucleon with respect to the deuteron and $\left|\phi_{3 \mathrm{~N}}\right\rangle$ is a state [antisymmetrized in the $(2,3)$ subsystem] representing the free motion of the three outgoing nucleons. Exclusive observables can be further integrated over suitable phasespace domains to arrive at the semiexclusive or inclusive observables.

Inclusive observables can be, however, also computed in a different way, without any resort to explicit final-state kinematics $[79,80]$. In inclusive calculations, where only the final energy $E$ of the nuclear system is known, one encounters the so-called response functions, which are defined through the following integral:

$R_{A B}^{\mathrm{inc}}=\sum_{m_{i}, m_{f}} \int d f \delta\left(E-E_{f}\right)\left\langle\Psi_{f}^{(-)}\left|j_{3 \mathrm{~N}}^{A}\right| \Psi\right\rangle\left(\left\langle\Psi_{f}^{(-)}\left|j_{3 \mathrm{~N}}^{B}\right| \Psi\right\rangle\right)^{*}$,

and depend, in general, on two components of the nuclear current operator, $A$ and $B$. In Eq. (2.41) $m_{i}$ and $m_{f}$ represent the whole sets of the initial and final spin magnetic quantum numbers, respectively, while the $d f$ integral denotes the sum and the integration over all final $3 \mathrm{~N}$ states with the energy $E$. Using closure, Eq. (2.41) can be rewritten as

$$
R_{A B}^{\mathrm{inc}}=\sum_{m_{i}} \int d f\left\langle\Psi\left|\left(j_{3 \mathrm{~N}}^{B}\right)^{\dagger} \delta(E-H) j_{3 \mathrm{~N}}^{A}\right| \Psi\right\rangle,
$$

where $H$ is again the full $3 \mathrm{~N}$ Hamiltonian and the $3 \mathrm{~N}$ bound state does not contribute to the $d f$ integration for $E>0$. Within the Faddeev scheme [79,80], $R_{A B}^{\text {inc }}$ can be expressed in terms of some auxiliary states as

$$
\begin{aligned}
R_{A B}^{\mathrm{inc}}= & \frac{1}{2 \pi i} \sum_{m_{i}}\left[\left\langle\Psi\left|\left(j_{3 \mathrm{~N}}^{A}\right)^{\dagger}\right| \Psi^{B}\right\rangle^{*}-\left\langle\Psi\left|\left(j_{3 \mathrm{~N}}^{B}\right)^{\dagger}\right| \Psi^{A}\right\rangle\right] \\
= & \frac{3}{2 \pi i} \sum_{m_{i}}\left\{\left\langle\Psi\left|\left[j^{A}(1)\right]^{\dagger} G_{0}(1+P)\right| V^{B}\right\rangle^{*}\right. \\
& \left.-\left\langle\Psi\left|\left[j^{B}(1)\right]^{\dagger} G_{0}(1+P)\right| V^{A}\right\rangle\right\},
\end{aligned}
$$

where in turn $(C=A, B)$

$$
\left|\Psi^{C}\right\rangle=G_{0}(1+P)\left|V^{C}\right\rangle .
$$

The state $\left|V^{C}\right\rangle$ obeys the Faddeev-type equation

$$
\begin{aligned}
\left|V^{C}\right\rangle= & \left(1+t_{23} G_{0}\right) j^{C}(1)|\Psi\rangle \\
& +\left[t G_{0} P+\left(1+t_{23} G_{0}\right) V^{(1)} G_{0}(1+P)\right]\left|V^{C}\right\rangle,
\end{aligned}
$$

with the same integral kernel as in the treatment of $3 \mathrm{~N}$ scattering [81]. Interestingly, the relation between the auxiliary 

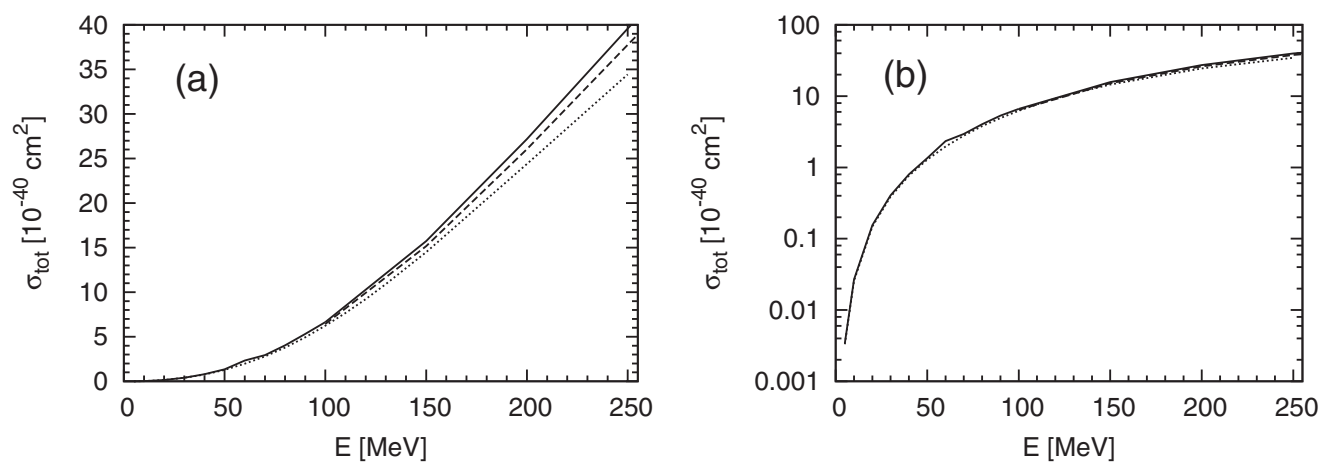

FIG. 2. The total cross section for the $v_{e}+{ }^{2} \mathrm{H} \rightarrow e^{-}+p+p$ reaction as a function of the initial neutrino energy $E$ shown on a linear scale (a) and on a logarithmic scale (b). The dashed (solid) line shows coordinate space predictions from Tables II and IV of Ref. [8] obtained with the AV18 potential and with the single-nucleon current (with the inclusion of single- and $2 \mathrm{~N}$ terms in the weak current operator), employing the relativistic kinematics. The dotted line displays results of nonrelativistic momentum space calculations from the present work obtained with the AV18 potential and with the single-nucleon current.

states defined in Eqs. (2.37) and (2.45) is very simple when the $3 \mathrm{~N}$ force is neglected:

$$
\left|V^{C}\right\rangle=j^{C}(1)|\Psi\rangle+\left|U^{C}\right\rangle
$$

The fact that $R_{A B}^{\mathrm{inc}}$ can be obtained by direct integrations or employing the method described above is used by us to test the numerical performance.

All the $3 \mathrm{~N}$ Faddeev-type equations are solved by iterations in the momentum-space basis

$$
|p q \alpha\rangle \equiv\left|p q(l s) j\left(\lambda \frac{1}{2}\right) I(j I) J m_{J}\left(t \frac{1}{2}\right) T m_{T}\right\rangle,
$$

which is an extension of the $(2,3)$ subsystem basis $\left|p \alpha_{2}\right\rangle$. Here $q$ is the magnitude of the Jacobi momentum, which describes the motion of the spectator nucleon 1 with respect to the center of mass of the $(2,3)$ subsystem. Consequently, the orbital angular momentum $\lambda$ of the spectator nucleon and its spin $\frac{1}{2}$ couple to the total spectator angular momentum $I$. The total angular momentum of the subsystem $(j)$ and the total angular momentum of nucleon $1(I)$ couple eventually to the total angular momentum of the $3 \mathrm{~N}$ system $J$ and its projection $m_{J}$. A corresponding coupling is introduced in the isospin space, where the $(2,3)$ total subsystem isospin $(t)$ together with the isospin of nucleon 1 builds the total $3 \mathrm{~N}$ isospin $T$ with the projection $m_{T}$. In practice, the calculations are restricted to a finite set of $\left|p \alpha_{2}\right\rangle$ and $|p \alpha\rangle$ states, which fulfill the condition $j \leqslant j_{\max }$ and $J \leqslant J_{\max }$. For the low $3 \mathrm{~N}$ internal energies and momentum transfers studied here convergence is achieved with $j_{\max }=3$ and $J_{\max }=\frac{15}{2}$.

In the following sections we describe our results, which are obtained, like in Ref. [8], without considering radiative corrections. Information about possible modifications of the results due to these effects are discussed in Ref. [52] and references cited therein.

\section{RESULTS FOR NEUTRINO SCATTERING $O{ }^{2} \mathrm{H}$}

Although on the way to calculate the total cross sections we evaluate the necessary integrands-the differential cross sections-we show here only the inclusive observables. For three selected reactions we compare our momentum-space results with the predictions from Shen et al. [8], which are also based on the traditional dynamical input. We restrict ourselves to the neutrino energies up to $250 \mathrm{MeV}$.

Let us start with the total cross section for the $v_{e}+{ }^{2} \mathrm{H} \rightarrow$ $e^{-}+p+p$ reaction, which is shown in Fig. 2 as a function of the initial neutrino energy $E$, both on a linear and on a logarithmic scale. The dashed and solid lines in this figure show the predictions from Tables II and IV of Ref. [8] obtained with the AV18 potential and with the single-nucleon current as well as with the single-nucleon current supplemented by the $2 \mathrm{~N}$ currents linked to the AV18 potential. The difference between these two curves highlights the importance of the $2 \mathrm{~N}$ currents for this reaction. The third, dotted, line is used to display results of our nonrelativistic calculations, carried out in momentum space with the AV18 potential and with the single-nucleon current. Here and in the following by "nonrelativistic calculations" we mean calculations that combine nonrelativistic nuclear dynamics with a nonrelativistic approach to nuclear kinematics. As mentioned, we use realtivistic formulas for lepton kinematics. Clearly, for the energies $E \leqslant 100 \mathrm{MeV}$, all three predictions essentially overlap, but for higher energies effects of $2 \mathrm{~N}$ contributions to the current operator are visible. The relativistic treatment of the nuclear kinematics by Shen et al. [8] leads to a clear spread between the dashed and the dotted line.

Elastic NC-driven (anti)neutrino scattering on ${ }^{2} \mathrm{H}$ has not been considered in Refs. [8,52]. It was studied, for example, by Frederico et al. [82] and later by Butler et al. [83], who investigated also the neutrino-deuteron break-up reactions within a $\chi$ EFT approach at next-to-leading order. The authors of Ref. [83] derived analytical expressions for the elastic (anti)neutrino-deuteron scattering cross section but did not yield direct results for the total cross sections. They were interested in the effects caused by the presence of the strange quarks in the deuteron. If the strangeness in the deuteron is neglected, then the results for the elastic channel are not only flavor independent but just the same for neutrino and antineutrino scattering. That is exactly the case for our calculations presented in Fig. 3. At the low (anti)neutrino energies considered here, this reaction is extremely hard to measure, due to the very small deuteron recoil energy. In addition, this reaction channel is strongly suppressed, as can be 

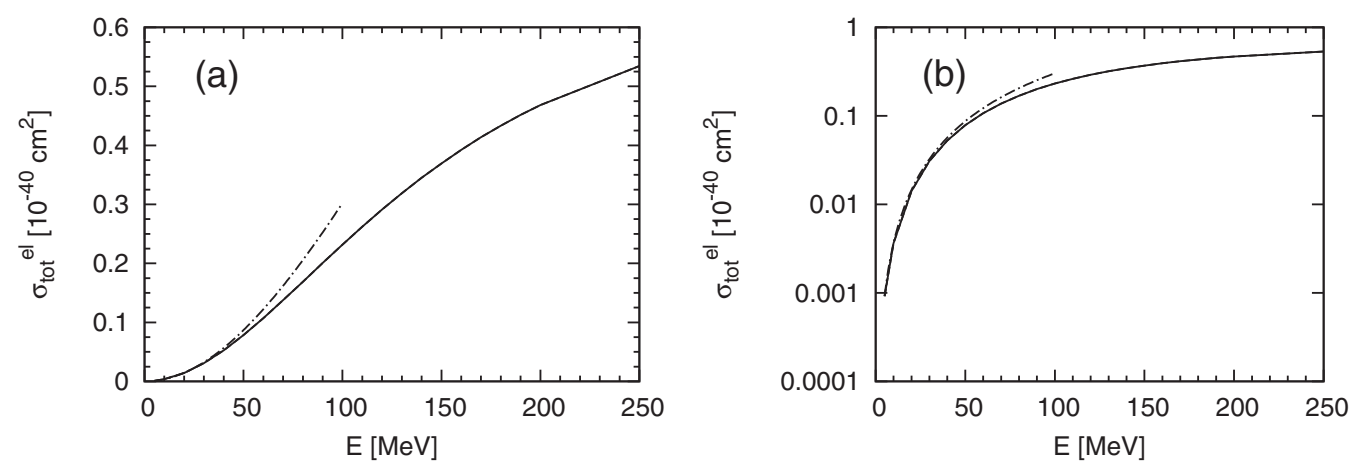

FIG. 3. The total cross section for the elastic NC (anti)neutrino scattering off the deuteron as a function of the initial (anti)neutrino energy $E$, shown on a linear scale (a) and on a logarithmic scale (b). The solid line displays results of nonrelativistic momentum space calculations from the present work obtained with the AV18 potential and with the single-nucleon current. These results are flavor independent and are the same for neutrino and antineutrino scattering. In addition, the dash-dotted line corresponds to the results from Butler et al. [83], ignoring the strangeness contents of the deuteron. See text for more details.

seen in Fig. 3, resulting in very small values of the total cross sections. In Fig. 3, beside our predictions, we show also the results derived from Eq. (34) in Ref. [83], setting the strange form factors to zero and calculating the $F_{C}$ form factor using the simple leading-order (LO) expression given by Eq. (31) in that reference. For low (anti)neutrino energies, where the LO formula is valid, both types of results nicely agree and clearly diverge in the higher-energy region.

The inelastic NC-induced reactions with the deuteron have been considered in Ref. [8], and in Fig. 4 we again compare results based on coordinate-space and momentum-space approaches for the antineutrino scattering. As in Fig. 2, we use results of Ref. [8], now from Tables II and III, for the total cross section for the $\bar{v}_{e}+{ }^{2} \mathrm{H} \rightarrow \bar{v}_{e}+p+n$ reaction. The solid and dashed curves represent results from Ref. [8] with and without $2 \mathrm{~N}$ contributions in the weak neutral nuclear current operator, accordingly. They have been calculated with the relativistic kinematics, so for higher energies in both figures the dashed lines visibly deviate from the dotted ones, representing our nonrelativistic momentum space predictions. These purely kinematical effects are of course essentially identical for the $\mathrm{CC}$ and NC-driven reactions with electron (anti)neutrinos, as the electron mass is very small compared to higher beam energies, where to a good approximation the electron could be treated as massless.

\section{RESULTS FOR NEUTRINO SCATTERING $\mathrm{ON}^{3} \mathrm{He}$ AND ${ }^{3} \mathrm{H}$}

The results presented in this section have also been obtained with the single-nucleon weak current operator from Refs. [8,49,63] (see Eqs. (2.9)-(2.10) in Ref. [63]) and with a nuclear Hamiltonian, which contains only the $2 \mathrm{~N}$ potential energy - the $3 \mathrm{~N}$ force has been neglected.

The same formalism which has been successfully developed for electron scattering and photodisintegration processes with $3 \mathrm{~N}$ systems $[79,80]$ as well as for muon capture on the $3 \mathrm{~N}$ bound states $[63,71]$ is directly applicable to elastic, quasielastic, and inelastic (anti)neutrino scattering on ${ }^{3} \mathrm{He}$ and ${ }^{3} \mathrm{H}$. The key elements of this formalism are presented in Sec. II.

First we consider the quasielastic electron antineutrino scattering on ${ }^{3} \mathrm{He}$, leading to the positron and ${ }^{3} \mathrm{H}$ nucleus in the final state. In this process, the same as in muon capture, weak CC operator changes the total charge of the nuclear
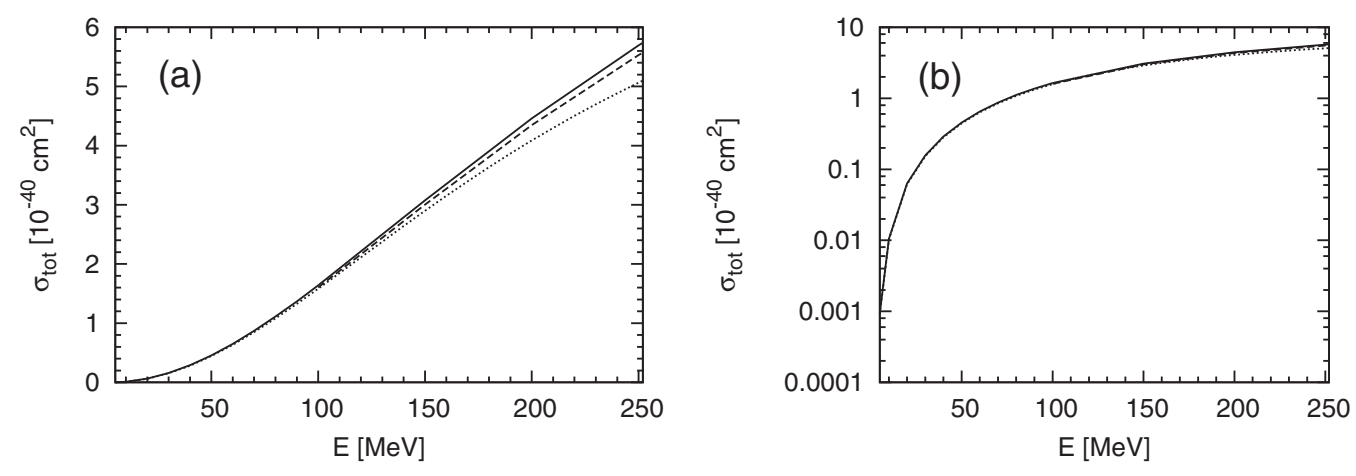

FIG. 4. The total cross section for the $\bar{v}_{e}+{ }^{2} \mathrm{H} \rightarrow \bar{v}_{e}+p+n$ reaction as a function of the initial antineutrino energy $E$ shown on a linear scale (a) and on a logarithmic scale (b). The dashed (solid) line shows coordinate space predictions from Tables II and III of Ref. [8] obtained with the AV18 potential and with the single-nucleon current (with the inclusion of single- and $2 \mathrm{~N}$ terms in the weak current operator), employing the relativistic kinematics. The dotted line displays results of nonrelativistic momentum space calculations from the present work obtained with the AV18 potential and with the single-nucleon current. 

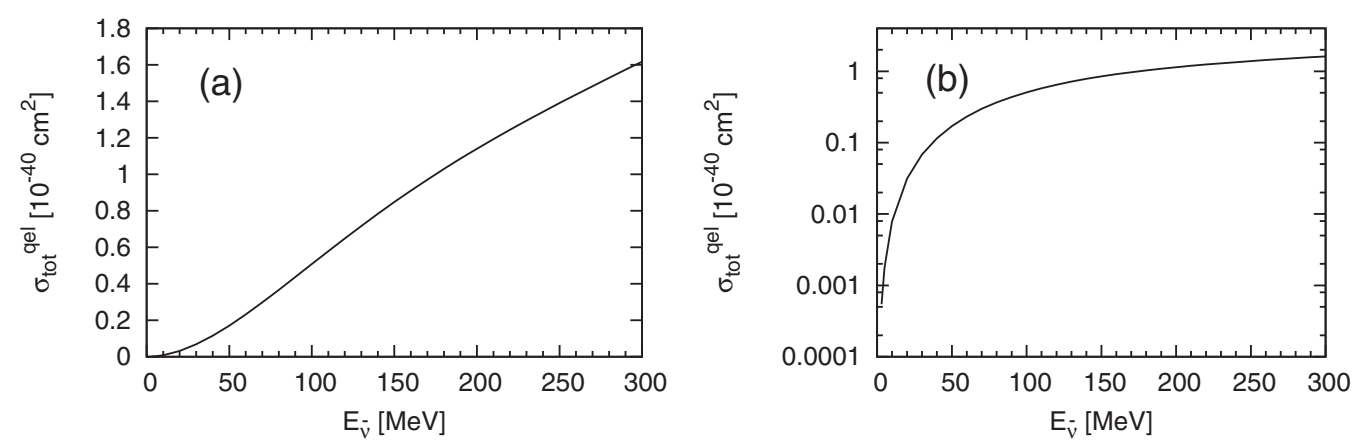

FIG. 5. The total cross section for the quasielastic $\mathrm{CC} \bar{\nu}_{e}+{ }^{3} \mathrm{He} \rightarrow e^{+}+{ }^{3} \mathrm{H}$ process as a function of the initial antineutrino energy $E$ shown on a linear scale (a) and on a logarithmic scale (b). The results are obtained with the AV18 NN potential and with the single-nucleon CC operator.

system. In Fig. 5 we show the total cross sections for the $\bar{v}_{e}+{ }^{3} \mathrm{He} \rightarrow e^{+}+{ }^{3} \mathrm{H}$ reaction as a function of the initial antineutrino energy.

Further non-break-up reactions with trinucleons are possible only with the neutral current. In Fig. 6 we display the predictions for the total cross section of the elastic $\bar{v}_{l}+{ }^{3} \mathrm{He} \rightarrow \bar{v}_{l}+{ }^{3} \mathrm{He}, \bar{v}_{l}+{ }^{3} \mathrm{H} \rightarrow \bar{v}_{l}+{ }^{3} \mathrm{H}, v_{l}+{ }^{3} \mathrm{He} \rightarrow v_{l}+$ ${ }^{3} \mathrm{He}$, and $v_{l}+{ }^{3} \mathrm{H} \rightarrow v_{l}+{ }^{3} \mathrm{H}$ reactions as a function of the initial (anti)neutrino energy. Despite very similar reaction kinematics, the cross sections take quite different values. The antineutrino- ${ }^{3} \mathrm{He}$ cross section is the smallest and the neutrino ${ }^{3} \mathrm{H}$ cross section reaches the highest values. Lines representing neutrino- ${ }^{3} \mathrm{He}$ and antineutrino- ${ }^{3} \mathrm{H}$ cross sections cross at $E \approx 150 \mathrm{MeV}$, with the former prevailing for the higher energies. All four predictions are flavor independent.

It is important to note that the difference between the predictions for the neutrino and antineutrino-induced reactions on each nucleus comes solely from the replacement of $V_{i j}$ functions from Eq. (2.24) by the $\bar{V}_{i j}$ functions defned in Eq. (2.25). In turn, the difference between the predictions for the $v_{l}+{ }^{3} \mathrm{He} \rightarrow v_{l}+{ }^{3} \mathrm{He}$ and $v_{l}+{ }^{3} \mathrm{H} \rightarrow v_{l}+{ }^{3} \mathrm{H}$ reactions is caused nearly exclusively by the proton $\left(j_{\mathrm{NC}}^{p}\right)$ and neutron $\left(j_{\mathrm{NC}}^{n}\right)$ contributions in the following isospin matrix element:

$$
\begin{aligned}
\mathcal{I}\left(t, m_{T}\right) \equiv & \left\langle\left(t \frac{1}{2}\right) \frac{1}{2} m_{T}\right| \frac{1}{2}\left[1+\tau_{z}(1)\right] j_{\mathrm{NC}}^{p} \\
& +\frac{1}{2}\left[1-\tau_{z}(1)\right] j_{\mathrm{NC}}^{n}\left|\left(t \frac{1}{2}\right) \frac{1}{2} m_{T}\right\rangle,
\end{aligned}
$$

which yield in the ${ }^{3} \mathrm{He}$ case $\left(m_{T}=\frac{1}{2}\right) \mathcal{I}\left(0, \frac{1}{2}\right)=j_{\mathrm{NC}}^{p}, \mathcal{I}\left(1, \frac{1}{2}\right)=$ $\frac{2}{3} j_{\mathrm{NC}}^{n}+\frac{1}{3} j_{\mathrm{NC}}^{p}$, and in the ${ }^{3} \mathrm{H}$ case $\left(m_{T}=-\frac{1}{2}\right) \mathcal{I}\left(0,-\frac{1}{2}\right)=j_{\mathrm{NC}}^{n}$, $\mathcal{I}\left(1,-\frac{1}{2}\right)=\frac{2}{3} j_{\mathrm{NC}}^{p}+\frac{1}{3} j_{\mathrm{NC}}^{n}$. The differences introduced by the slightly different masses and wave functions of ${ }^{3} \mathrm{He}$ and ${ }^{3} \mathrm{H}$ are practically negligible. The same is also true for the two antineutrino-induced elastic reactions.

The CC- and NC-driven break-up reactions are definitely more demanding than the formerly discussed ones due to the complicated kinematics, which has to take into account twoand three-body disintegration processes. Full inclusion of the final-state interactions is even more challenging, especially for the isospin-raising reactions-induced by the neutrinos, for which two or three outgoing nucleons are charged.

In the studies of electron scattering on the trinucleons one usually assumes that the initial electron energy, the electron scattering angle and the final electron energy are known. This information allows one to study the $3 \mathrm{~N}$ scattering states at a fixed $3 \mathrm{~N}$ internal energy and at a fixed total $3 \mathrm{~N}$ momentum. In the case of the (anti)neutrino induced processes one is interested predominantly in the total cross sections, which necessitates a calculation of at least hundreds of the neutrino kinematics. Even for the (anti)neutrino reactions with the deuteron calculations are indeed time consuming.

It is then important to realize that the essential dynamical quantities for inclusive reactions, the so-called response functions $R_{i}$, depend on two parameters only. These parameters
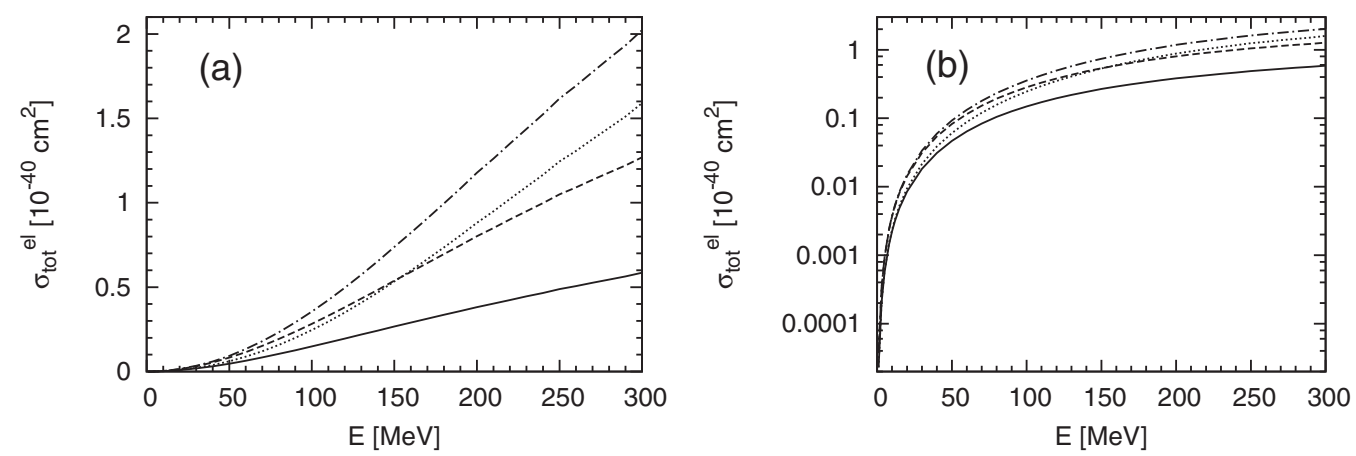

FIG. 6. The total cross section for the elastic NC-driven reactions $\bar{v}_{l}+{ }^{3} \mathrm{He} \rightarrow \bar{v}_{l}+{ }^{3} \mathrm{He}$ (solid line), $\bar{v}_{l}+{ }^{3} \mathrm{H} \rightarrow \bar{v}_{l}+{ }^{3} \mathrm{H}($ dashed line), $v_{l}+{ }^{3} \mathrm{He} \rightarrow v_{l}+{ }^{3} \mathrm{He}$ (dotted line), and $v_{l}+{ }^{3} \mathrm{H} \rightarrow v_{l}+{ }^{3} \mathrm{H}$ (dash-dotted line) as a function of the initial (anti)neutrino energy $E$ shown on a linear scale (a) and a logarithmic scale (b). The results are obtained with the AV18 NN potential and the single-nucleon NC operator. 

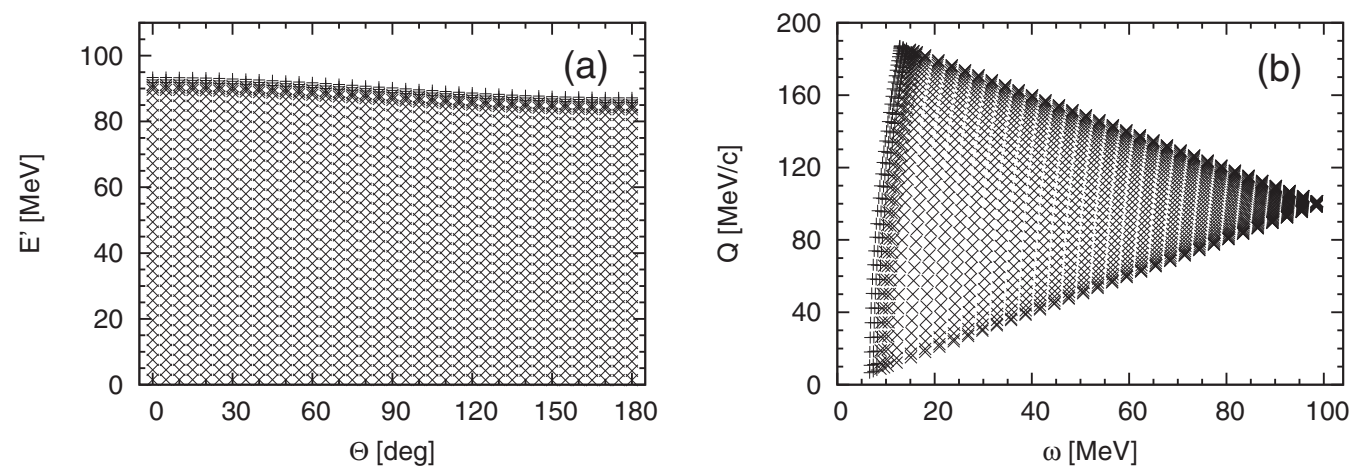

FIG. 7. The ranges of various kinematical quantities describing the kinematics of the NC-driven break-up of ${ }^{3} \mathrm{H}$ for the initial (anti)neutrino energy $E=100 \mathrm{MeV}$. In (a) the outgoing (anti)neutrino energy and the scattering angle are considered, while in (b) the magnitude of the three-momentum transfer is plotted versus the energy transfer.
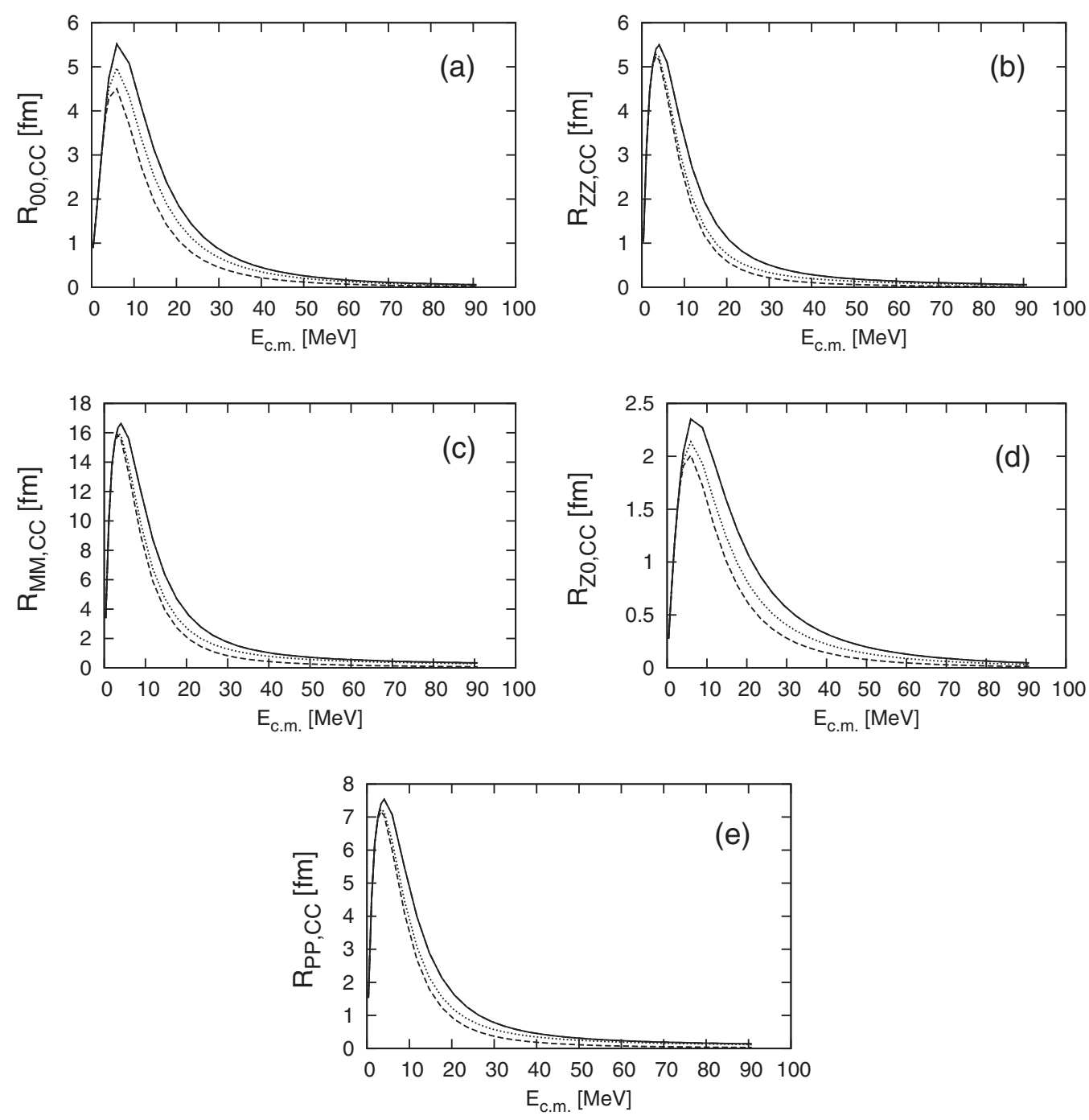

FIG. 8. The inclusive CC response functions $R_{00, \mathrm{CC}}(\mathrm{a}), R_{\mathrm{ZZ}, \mathrm{CC}}(\mathrm{b}), R_{\mathrm{MM}, \mathrm{CC}}(\mathrm{c}), R_{\mathrm{Z} 0, \mathrm{CC}}(\mathrm{d})$ and $R_{\mathrm{PP}, \mathrm{CC}}(\mathrm{e})$ for the electron antineutrino disintegration of ${ }^{3} \mathrm{He}$ as a function of the internal $3 \mathrm{~N}$ energy $E_{\text {c.m. }}$ for the fixed value of the three-momentum transfer $Q=100 \mathrm{MeV} / c$. The results are obtained with the AV18 NN potential and the single-nucleon CC operator. The dotted line shows the contribution from final nuclear states with the total isospin $T=\frac{1}{2}$ and the dash-dotted line represents the total response function obtained from the total isospin $T=\frac{1}{2}$ and $T=\frac{3}{2}$ states. The dashed line depicts the part of the response function stemming from the two-body break-up channel and the solid line the total response function, receiving contributions from both two- and three-body break-up states. Note that the dash-dotted and solid lines overlap. 

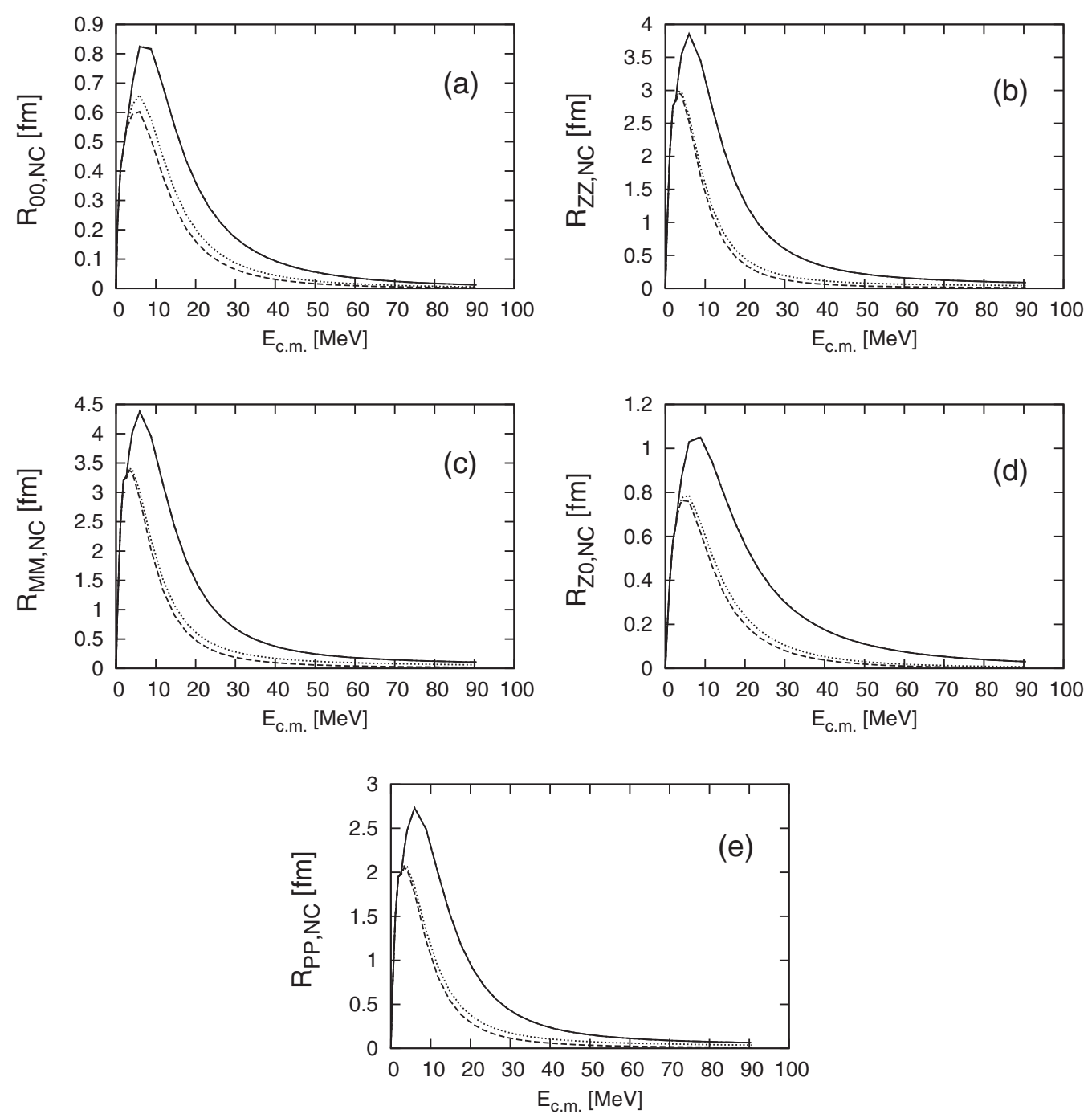

FIG. 9. The inclusive NC response functions $R_{00, \mathrm{NC}}(\mathrm{a}), R_{\mathrm{ZZ}, \mathrm{NC}}(\mathrm{b}), R_{\mathrm{MM}, \mathrm{NC}}(\mathrm{c}), R_{\mathrm{Z} 0, \mathrm{NC}}$ (d), and $R_{\mathrm{PP}, \mathrm{NC}}(\mathrm{e})$ for the (anti)neutrino disintegration of ${ }^{3} \mathrm{H}$ as a function of the internal $3 \mathrm{~N}$ energy $E_{\text {c.m. }}$ for the fixed value of the three-momentum transfer $Q=100 \mathrm{MeV} / c$. The results are obtained with the AV18 NN potential and the single-nucleon NC operator. Lines as in Fig. 8.

are the energy transfer $\omega$ and the magnitude of the threemomentum transfer $Q \equiv|\mathbf{Q}|$. We show in Fig. 7 the ranges of these quantities for the $\mathrm{NC}$ neutrino inelastic scattering for the initial (anti)neutrino energy $E=100 \mathrm{MeV}$. Of course the same statement is true also for the CC-induced reactions, so in both cases the total cross sections are built from the purely kinematical input and the response functions, calculated in the whole physical $\omega-Q$ region.

The response functions $R_{i} \equiv R_{i}(\omega, Q)$ stem from various products of the nuclear matrix elements: $R_{00} \propto\left|N^{0}\right|^{2}, R_{Z Z} \propto$ $\left|N_{z}\right|^{2}, \quad R_{M M} \propto\left|N_{-1}\right|^{2}, \quad R_{Z 0} \propto \operatorname{Re}\left(N^{0}\left(N_{z}\right)^{\star}\right), \quad$ and $R_{P P} \propto$ $\left|N_{+1}\right|^{2}$, receiving contributions from all the final nuclear states. Using the approach described in Eq. (2.43), these contributions can be separately calculated for the two values of the total $3 \mathrm{~N}$ isospin, $T=\frac{1}{2}$ and $T=\frac{3}{2}$. On the other hand, the same sum over the final nuclear states can be performed over the physical two-body and three-body fragmentation channels. An agreement between these two approaches provides a nontrivial test of numerics.
In this work we restrict ourselves to a sample of results for the $\mathrm{CC}$ and $\mathrm{NC}$ response functions. They are calculated for the fixed value of the three-momentum transfer $Q=100 \mathrm{MeV} / c$ as a function of the internal $3 \mathrm{~N}$ energy $E_{\mathrm{c} . \mathrm{m}}$. The latter quantity is simply related to the energy transfer. For example, in the case of the antineutrino $\mathrm{CC}$ break-up of ${ }^{3} \mathrm{H}$ it reads

$$
E_{\text {c.m. }}=\omega+M_{3^{3}}-3 M_{n}-\frac{\mathbf{Q}^{2}}{6 M_{n}},
$$

where the $M_{3_{\mathrm{H}}}$ and $M_{n}$ are the triton and neutron masses, respectively.

The five inclusive CC response functions $R_{i, \mathrm{CC}}$ for the electron antineutrino disintegration of ${ }^{3} \mathrm{He}$ are shown in Fig. 8. They all have a very similar shape, known also from inclusive electron-nucleus scattering (see, for example, Ref. [84]): They start from zero at threshold, rise to reach a maximum, whose position corresponds to antineutrino scattering elastically from a moving bound nucleon, and slowly tend to zero for higher $E_{\text {c.m. }}$ values. In Fig. 8 we show also separate contributions 

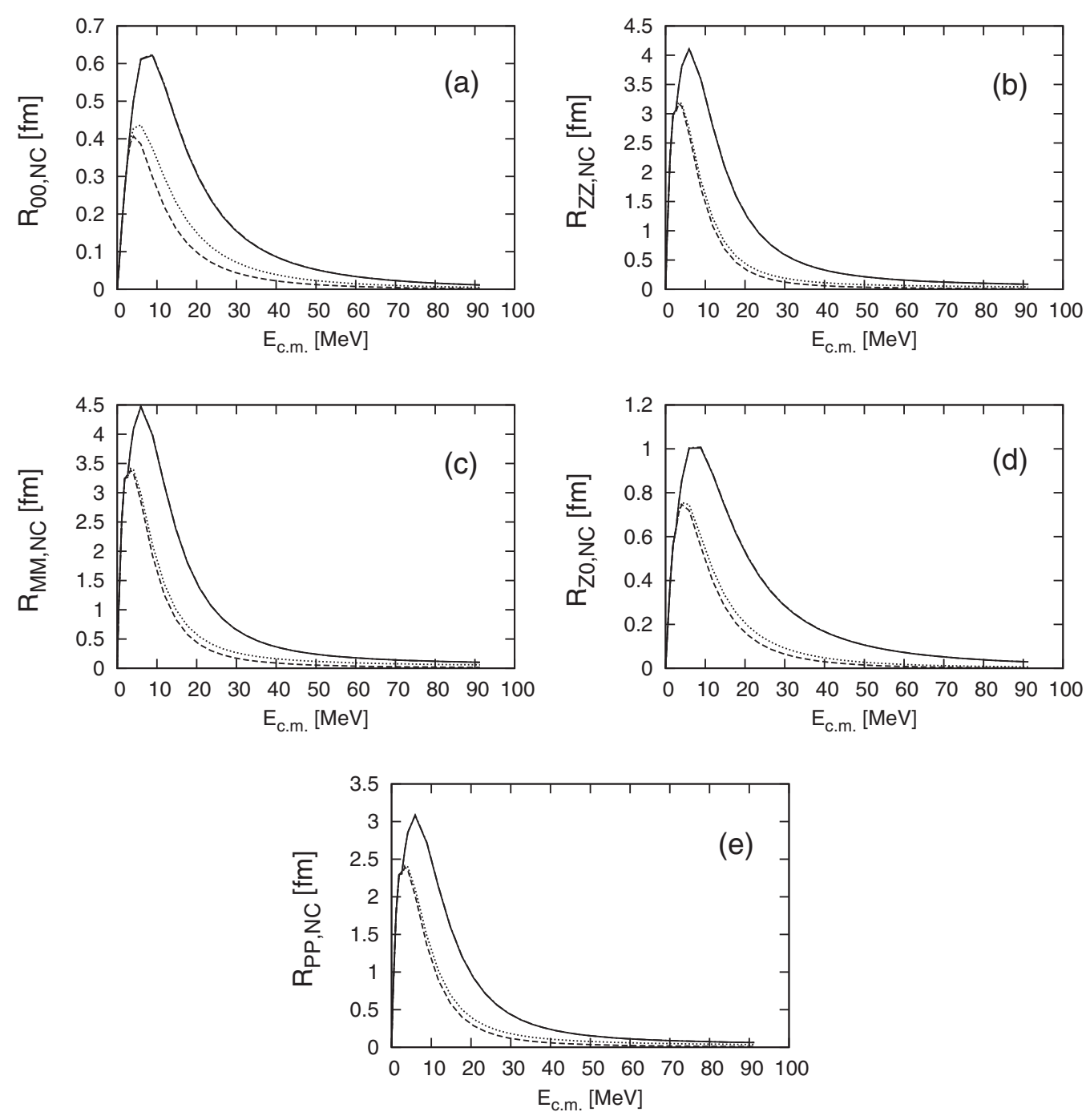

FIG. 10. The same as in Fig. 9 for the (anti)neutrino disintegration of ${ }^{3} \mathrm{He}$.

from the total isospin $T=\frac{1}{2}$ states (dotted line) and from the two-body break-up channel (dashed line), while the total response function is computed either as a sum of the $T=\frac{1}{2}$ and $T=\frac{3}{2}$ parts (dash-dotted line) or as a sum over the two- and three-body break-up contributions (solid line). As expected, for each $R_{i}$ the dash-dotted and solid lines overlap and the relative distance between the solid and dotted lines provides information about the importance of the $T=\frac{3}{2}$ (present only in the three-body break-up) contribution. The corresponding difference between the solid and dashed lines provides information about the contribution to the total response function from the three-body break-up channel.

In the same way we display the inclusive $\mathrm{NC}$ response functions $R_{i, \mathrm{NC}}$ for the (anti)neutrino disintegration of ${ }^{3} \mathrm{H}$ in Fig. 9 and for the (anti)neutrino disintegration of ${ }^{3} \mathrm{He}$ in Fig. 10. These response functions are the same for the neutrino and antineutrino-induced reactions. From Figs. 9 and 10 we infer that both the $T=\frac{3}{2}$ and the three-body break-up contributions to the inclusive NC response functions are relatively much more important than for the $\mathrm{CC}$ response functions. Our predictions for the (anti)neutrino disintegration of ${ }^{3} \mathrm{He}$ suffer from the lack of Coulomb force in the calculations of the final nuclear states. Our experience from investigations of electron scattering on ${ }^{3} \mathrm{He}$ tells us, however, that the Coulomb force does not substantially affect inclusive response functions. Within our present framework we cannot describe CC neutrinoinduced processes with three protons in the final state.

\section{SUMMARY AND CONCLUSIONS}

A consistent framework for the calculations of several neutrino-induced processes on ${ }^{2} \mathrm{H},{ }^{3} \mathrm{He},{ }^{3} \mathrm{H}$ and other light nuclei is still a challenge, despite recent progress in this field. There are many models of the nuclear interactions and weak current operators linked to these forces, but full compatibility has not been achieved yet. The work on the regularization of the chiral potentials and electroweak current operators is in progress and the crucial issue of the current conservation has to be ultimately solved. Additional problems arise from the fact that (anti)neutrinos can transfer a lot of energy and threemomentum to the nuclear system which requires a relativistic treatment of both kinematics and dynamics. 
In the present paper we studied selected two- and threenucleon reactions with (anti)neutrinos in the framework close to the one of Ref. [8] but with the single-nucleon current operator. For most of the reactions we provided predictions for the total cross sections. In the case of the (anti)neutrino${ }^{3} \mathrm{He}$ and (anti)neutrino- ${ }^{3} \mathrm{H}$ inelastic scattering, we computed examples of the essential response functions.

Our calculations for the reactions with the deuteron were a necessary test before embarking on $3 \mathrm{~N}$ calculations, although, contrary to Ref. [8], we restricted ourselves to the lower (anti)neutrino energies. But even in this more restricted range of neutrino energies relativistic effects in the kinematics were thoroughly checked with the result that the main difference between the relativistic and nonrelativistic kinematics stemmed from the form of the phase-space factor. We worked exclusively in momentum space, treating also the Coulomb interaction between the two outgoing protons in the form of a sharply cut off potential. This is justified because current matrix elements become negligible for sufficiently large distances between two nucleons. Last but not least, very important elements of the formalism- $2 \mathrm{~N}$ scattering states in the partial wave representation-were cross checked using the results from the momentum and coordinate space calculations.

The full understanding of the studied (anti)neutrino-induced processes requires the inclusion of at least $2 \mathrm{~N}$ contributions to the nuclear current operators and, especially at larger (anti)neutrino energies, consistent relativistic treatment of kinematics and dynamics. Note that momentum space offers an easier possibility to use the "boosted potential," which has been already employed in various relativistic studies of few-nucleon systems [64-70]. We plan to work along this line and perform more complete calculations in the near future, ultimately with chiral dynamical input. We believe, however, that the predictions presented here can serve as an important benchmark.

\section{ACKNOWLEDGMENTS}

This work is a part of the LENPIC project and was supported by the Polish National Science Center under Grants No. 2016/22/M/ST2/00173 and No. 2016/21/D/ST2/01120. The numerical calculations were partially performed on the supercomputer cluster of the JSC, Jülich, Germany.
[1] K. Kubodera and S. Nozawa, Int. J. Mod. Phys. E 3, 101 (1994).

[2] S. Nakamura, T. Sato, V. Gudkov, and K. Kubodera, Phys. Rev. C 63, 034617 (2001); 73, 049904(E) (2006).

[3] S. Nakamura et al., Nucl. Phys. A 707, 561 (2002).

[4] J. Carlson and R. Schiavilla, Rev. Mod. Phys. 70, 743 (1998).

[5] R. B. Wiringa, V. G. J. Stoks, and R. Schiavilla, Phys. Rev. C 51, 38 (1995).

[6] R. Machleidt, Phys. Rev. C 63, 024001 (2001).

[7] Q. R. Ahmad et al. (SNO Collaboration), Phys. Rev. Lett. 89, 011301 (2002); S. N. Ahmed et al. (SNO Collaboration), ibid. 92, 181301 (2004).

[8] G. Shen, L. E. Marcucci, J. Carlson, S. Gandolfi, and R. Schiavilla, Phys. Rev. C 86, 035503 (2012).

[9] M. Butler, J.-W. Chen, and X. Kong, Phys. Rev. C 63, 035501 (2001).

[10] S. Weinberg, Phys. Lett. B 251, 288 (1990).

[11] S. Weinberg, Nucl. Phys. B 363, 3 (1991).

[12] E. Epelbaum, W. Glöckle, and U.-G. Meißner, Nucl. Phys. A 637, 107 (1998); 671, 295 (2000).

[13] E. Epelbaum, W. Glöckle, and U.-G. Meißner, Eur. Phys. J. A 19, 125 (2004).

[14] E. Epelbaum, W. Glöckle, and U.-G. Meißner, Nucl. Phys. A 747, 362 (2005)

[15] E. Epelbaum, A. Nogga, W. Glockle, H. Kamada, Ulf-G. Meissner, and H. Witala, Phys. Rev. C 66, 064001 (2002).

[16] E. Epelbaum, Prog. Part. Nucl. Phys. 57, 654 (2006).

[17] E. Epelbaum, H.-W. Hammer, and U.-G. Meißner, Rev. Mod. Phys. 81, 1773 (2009).

[18] V. Bernard, E. Epelbaum, H. Krebs, and Ulf-G. Meißner, Phys. Rev. C 77, 064004 (2008).

[19] V. Bernard, E. Epelbaum, H. Krebs, and Ulf-G. Meißner, Phys. Rev. C 84, 054001 (2011).

[20] E. Epelbaum, Eur. Phys. J. A 34, 197 (2007).

[21] H. Krebs and E. Epelbaum, Few Body Syst. 50, 295 (2011).
[22] H. Krebs, A. M. Gasparyan, and E. Epelbaum, arXiv:1803.09613 [nucl-th].

[23] E. Epelbaum, H. Krebs, and U.-G. Meißner, Eur. Phys. J. A 51, 26 (2015).

[24] E. Epelbaum, H. Krebs, and U.-G. Meißner, Phys. Rev. Lett. 115, 122301 (2015).

[25] S. Binder, A. Calci, E. Epelbaum, R. J. Furnstahl, J. Golak, K. Hebeler, H. Kamada, H. Krebs, J. Langhammer, S. Liebig, P. Maris, Ulf-G. Meißner, D. Minossi, A. Nogga, H. Potter, R. Roth, R. Skibiński, K. Topolnicki, J. P. Vary, and H. Witała, Phys. Rev. C 93, 044002 (2016).

[26] R. Skibiński, J. Golak, K. Topolnicki, H. Witała, E. Epelbaum, H. Krebs, H. Kamada, Ulf-G. Meißner, and A. Nogga, Phys. Rev. C 93, 064002 (2016).

[27] P. Reinert, H. Krebs, and E. Epelbaum, Eur. Phys. J. A 54, 86 (2018).

[28] R. Navarro Pérez, J. E. Amaro, and E. R. Arriola, Phys. Rev. C 88, 064002 (2013); 91, 029901(E) (2015).

[29] S. Kölling, E. Epelbaum, H. Krebs, and U.-G. Meißner, Phys. Rev. C 80, 045502 (2009).

[30] S. Kölling, E. Epelbaum, H. Krebs, and U.-G. Meißner, Phys. Rev. C 84, 054008 (2011).

[31] H. Krebs, E. Epelbaum, and U.-G. Meißner, Ann. Phys. 378, 317 (2017).

[32] D. Rozpędzik, J. Golak, S. Kölling, E. Epelbaum, R. Skibiński, H. Witała, and H. Krebs, Phys. Rev. C 83, 064004 (2011).

[33] R. Skibiński, J. Golak, D. Rozpędzik, K. Topolnicki, and H. Witała, Acta Phys. Polon. B 46, 159 (2015).

[34] D. R. Entem and R. Machleidt, Phys. Rev. C 68, 041001(R) (2003).

[35] R. Machleidt and D. R. Entem, Phys. Rep. 503, 1 (2011).

[36] D. R. Entem, R. Machleidt, and Y. Nosyk, Phys. Rev. C 96, 024004 (2017).

[37] T.-S. Park, D.-P. Min, and M. Rho, Phys. Rep. 233, 341 (1993). 
[38] T.-S. Park, D.-P. Min, and M. Rho, Nucl. Phys. A 596, 515 (1996).

[39] S. Pastore, R. Schiavilla, and J. L. Goity, Phys. Rev. C 78, 064002 (2008).

[40] S. Pastore, L. Girlanda, R. Schiavilla, M. Viviani, and R. B. Wiringa, Phys. Rev. C 80, 034004 (2009).

[41] S. Pastore, L. Girlanda, R. Schiavilla, and M. Viviani, Phys. Rev. C 84, 024001 (2011).

[42] A. Baroni, L. Girlanda, S. Pastore, R. Schiavilla, and M. Viviani, Phys. Rev. C 93, 015501 (2016); 93, 049902(E) (2016); 95, 059901(E) (2017).

[43] M. Piarulli, L. Girlanda, L. E. Marcucci, S. Pastore, R. Schiavilla, and M. Viviani, Phys. Rev. C 87, 014006 (2013).

[44] D. Gazit, S. Quaglioni, and P. Navratil, Phys. Rev. Lett. 103, 102502 (2009).

[45] A. Baroni, L. Girlanda, A. Kievsky, L. E. Marcucci, R. Schiavilla, and M. Viviani, Phys. Rev. C 94, 024003 (2016); 95, 059902(E) (2017).

[46] T.-S. Park, L. E. Marcucci, R. Schiavilla, M. Viviani, A. Kievsky, S. Rosati, K. Kubodera, D.-P. Min, and M. Rho, Phys. Rev. C 67, 055206 (2003).

[47] L. E. Marcucci, R. Schiavilla, and M. Viviani, Phys. Rev. Lett. 110, 192503 (2013).

[48] D. Gazit, Phys. Lett. B 666, 472 (2008).

[49] L. E. Marcucci, M. Piarulli, M. Viviani, L. Girlanda, A. Kievsky, S. Rosati, and R. Schiavilla, Phys. Rev. C 83, 014002 (2011).

[50] L. E. Marcucci, A. Kievsky, S. Rosati, R. Schiavilla, and M. Viviani, Phys. Rev. Lett. 108, 052502 (2012).

[51] L. E. Marcucci and R. Machleidt, Phys. Rev. C 90, 054001 (2014).

[52] A. Baroni and R. Schiavilla, Phys. Rev. C 96, 014002 (2017).

[53] D. Gazit and N. Barnea, Phys. Rev. C 70, 048801 (2004).

[54] D. Gazit and N. Barnea, Phys. Rev. Lett. 98, 192501 (2007).

[55] E. O'Connor, D. Gazit, C. J. Horowitz, A. Schwenk, and N. Barnea, Phys. Rev. C 75, 055803 (2007).

[56] D. Efros, W. Leidemann, and G. Orlandini, Phys. Lett. B 338, 130 (1994).

[57] N. Barnea, W. Leidemann, and G. Orlandini, Phys. Rev. C 61, 054001 (2000); Nucl. Phys. A 693, 565 (2001).

[58] N. Barnea and A. Novoselsky, Ann. Phys. (NY) 256, 192 (1997).

[59] A. Lovato, S. Gandolfi, J. Carlson, S. C. Pieper, and R. Schiavilla, Phys. Rev. Lett. 112, 182502 (2014).

[60] A. Lovato, S. Gandolfi, J. Carlson, S. C. Pieper, and R. Schiavilla, Phys. Rev. C 91, 062501(R) (2015).

[61] A. S. Meyer, M. Betancourt, R. Gran, and R. J. Hill, Phys. Rev. D 93, 113015 (2016).
[62] K. Topolnicki, J. Golak, R. Skibiński, A. E. Elmeshneb, W. Glöckle, A. Nogga, and H. Kamada, Few Body Syst. 54, 2233 (2013).

[63] J. Golak, R. Skibiński, H. Witała, K. Topolnicki, A. E. Elmeshneb, H. Kamada, A. Nogga, and L. E. Marcucci, Phys. Rev. C 90, 024001 (2014).

[64] H. Kamada, W. Glöckle, J. Golak, and Ch. Elster, Phys. Rev. C 66, 044010 (2002).

[65] H. Witała, J. Golak, W. Glöckle, and H. Kamada, Phys. Rev. C 71, 054001 (2005).

[66] J. Golak, R. Skibiński, H. Witała, W. Glöckle, A. Nogga, and H. Kamada, Acta Phys. Polon. B 38, 2143 (2006).

[67] T. Lin, Ch. Elster, W. N. Polyzou, H. Witała, and W. Glöckle, Phys. Rev. C 78, 024002 (2008).

[68] H. Witała, J. Golak, R. Skibiński, W. Glöckle, H. Kamada, and W. N. Polyzou, Phys. Rev. C 83, 044001 (2011); 88, 069904(E) (2013).

[69] W. N. Polyzou, Y. Huang, Ch. Elster, W. Glöckle, J. Golak, R. Skibiński, H. Witała, and H. Kamada, Few Body Syst. 49, 129 (2011).

[70] W. N. Polyzou and Ch. Elster, J. Phys. G 41, 094006 (2014).

[71] J. Golak, R. Skibiński, H. Witała, K. Topolnicki, H. Kamada, A. Nogga, and L. E. Marcucci, Phys. Rev. C 94, 034002 (2016).

[72] J. D. Walecka, Theoretical Nuclear and Subnuclear Physics (Oxford University Press, New York, 1995).

[73] J. D. Bjorken and S. D. Drell, Relativistic Quantum Mechanics (McGraw-Hill, New York, 1998).

[74] C. Lanczos, J. Math. Phys. 17, 123 (1938).

[75] C. de Boor and B. Swartz, SIAM J. Numer. Anal. 10, 582 (1973).

[76] N. W. Schellingerhout, Ph.D. thesis, Rijksuniversiteit Groningen, The Netherlands, 1995.

[77] E. Fermi, Z. Phys. 88, 161 (1934).

[78] A. Nogga, D. Hüber, H. Kamada, and W. Glöckle, Phys. Lett. B 409, 19 (1997).

[79] J. Golak, R. Skibiński, H. Witała, W. Glöckle, A. Nogga, and H. Kamada, Phys. Rept. 415, 89 (2005).

[80] S. Ishikawa, J. Golak, H. Witała, H. Kamada, W. Glöckle, and D. Hüber, Phys. Rev. C 57, 39 (1998).

[81] D. Hüber, H. Kamada, H. Witała, and W. Glöckle, Acta Phys. Pol. B 28, 1677 (1997).

[82] T. Frederico, E. M. Henley, S. J. Pollock, and S. Ying, Phys. Rev. C 46, 347 (1992).

[83] M. Butler and J.-W. Chen, Nucl. Phys. A 675, 575 (2000).

[84] O. Benhar, D. Day, and I. Sick, Rev. Mod. Phys. 80, 189 (2008). 